\title{
Mitigation of installation-related effects for small-scale borehole-to-surface ERT
}

\author{
Johanna Ochs* Norbert Klitzsch* Florian M. Wagner*
}

This is the authors' peer-reviewed and accepted preprint of a paper published in the Journal of Applied Geophysics. It contains minor differences to the publisher's version. The published paper can be found here: https://doi.org/10.1016/j.jappgeo.2022.104530

*Institute for Applied Geophysics and Geothermal Energy, RWTH Aachen University, Mathieustraße 30, 52074 Aachen, Germany; Contact: jochs@eonerc.rwth-aachen.de 


\section{Abstract}

Small-scale resistivity inhomogeneities can result from the local distribution of water and the water and nutrient uptake of plants. Measuring small-scale Electrical Resistivity Tomography (ERT) in the field comes with a set of particularities, especially when including borehole electrodes for a better resolution with depth. We apply small-scale borehole-to-surface ERT over a palaeochannel. Combining surface ERT with detailed borehole-to-surface ERT profiles along the measurement line allows a delineation of finer layering within the coarser lithology. Our field setup includes a borehole electrode tool with 20 ring electrodes, electrically coupled to the ground via a conductive mud. Two main points are addressed in this publication: 1 . In the field, we electrically coupled the borehole electrodes to the ground by filling the cavities around the tool with a soil mud, i.e., we need to account for the unknown conductive borehole filling in the inversion. If not incorporated, the mud has a considerable influence on the resistivities close to the borehole tool, but also on the region around the surface electrodes. Consequently, alongside with a $3 \mathrm{D}$ inversion scheme representing the electrodes with the Complete Electrode Model (CEM), we include the mud as a separate and uncoupled region. We model the geometry of the mud layer around the tool and do not allow an influence of this region on the rest of the model. 2. Due to the small electrode distances and the overall small-scale nature of the array, the depth of installation of the borehole electrode tool must be known accurately in the inversion model. However, it is not easy to measure the tool depth in the field with the required accuracy, due to small-scale surface roughness, e.g., from a weathered loose soil layer at the surface or from

vegetation. We also investigate the influence of a tilted tool installation and optimise for the depth and installation angle of the borehole tool before inverting for resistivities. An accurate knowledge of the borehole electrode positions is crucial for a reliable and precise inversion result. The surface electrodes establish a coordinate system around the borehole tool on the surface, with an angle $\varphi$ describing the direction around the tool in the top view. The sensitive plane (in-plane) is defined as the $\mathrm{X}-\mathrm{z}$ plane cutting through $\varphi=0^{\circ}$ and $\varphi=180^{\circ}$. A tilting of the tool from the vertical direction is described by a tilting angle $\theta$. A tilting of the borehole tool within the sensitive plane manifests in an increased misfit between data points on both sides of the tool, i.e., at $\varphi=0^{\circ}$ and $\varphi=180^{\circ}$. We use this difference to optimise on the tool angle. The true depth of the borehole tool is found by searching for a minimum of the objective function, describing the goodness of the found model, while assuming different tool depths in each inversion. We see a minimum of the objective function, which can be attributed to the correct depth range, as shown by a synthetic study. Through our optimisations, we can determine a tilting of the tool, i.e., the angle $\theta$, with an accuracy of $2^{\circ}$ to $3^{\circ}$ and the tool depth with an accuracy of a few centimetres, depending partially on the subsurface resistivities, i.e., our optimisation works mainly in predominantly horizontally layered soils. A tilting in directions out of the sensitive plane (out-of-plane) can be projected onto the sensitive plane, since the out-of-plane tilt has a negligible influence on the data. After this optimisation, we can determine layer resistivities from our field data.

Keywords: small-scale geoelectrics, complete electrode model, borehole-to-surface ERT, installation effects, borehole electrode positions

\section{Introduction}

Field- to plot-scale soil heterogeneity is an important factor for the availability and transport of water and nutrients, but also for the distribution of contaminants (see e.g. Pätzold et al., 2008). Detailed knowledge about this heterogeneity can be used in precision agriculture and to parametrise local flow models. There exists a close link between weed growth and soil heterogeneity (Pätzold et al., 2020). 
Local heterogeneity also plays an important role in Earth System Models, e.g., regarding infiltration-runoff partitioning and recharge in vegetated regions (Fatichi et al., 2020), and can also be integrated in a stochastic manner for accurate water flow modelling (Nezhad et al., 2011).

The influence of even smaller heterogeneity on vertical transport, like thin clayey or gravelly layers on the scale of a few $\mathrm{cm}$, and their detection, has been the subject of few studies. Kollet (2009) proposes an up-scaling and averaging approach for sub-grid scale soil heterogeneity, i.e., smaller than the resolution of the model grid, in coupled groundwater flow models. According to the author, soil heterogeneity plays an important role especially in dry periods, but is negligible for water availability in wet periods. The author also points out that a detection of small-scale soil heterogeneity is difficult and usually not implemented in flow models. Studies working on this scale often rely on soil sampling, like in Rosenfeld et al. (2017), who investigate soils on a microscopic scale, or applied to urban soil heterogeneity, as described in Greinert $(2015)$.

While helpful in determining the ground truth, with soil sampling, thin layers can be missed and remain unsampled. With geophysical methods however, we can image the subsurface. Therefore, a combination of both approaches is ideal for accurately depicting the subsurface. In the context of soil water, Vanella et al. (2018) show the feasibility of time-lapse small-scale 3D Electrical Resistivity Tomography (ERT) for monitoring the soil-root interaction and root water uptake at the $\mathrm{cm}$ scale and investigate the influence of different irrigation regimes on tree roots. They utilise superficial, buried, and borehole electrodes for their measurements.

The detection of small-scale water barriers with geophysical methods solely from the surface is desirable but difficult and requires a high resolution at the depth of interest. Geophysical imaging methods such as ERT usually suffer from a decrease of resolution with depth, making it difficult to accurately image the boundaries of very thin layers (see e.g. Ochs \& Klitzsch $(\overline{2020})$ and references therein). Also, highly conductive layers directly at the surface followed by less conductive material below can focus the current in this topmost layer and reduce the current density and therefore sensitivity in lower layers.

A common problem specifically in small-scale ERT is the influence of the rather large electrodes in comparison with the electrode spacing. There are different approaches for minimizing their effect. Verdet et al. (2018) approximate the finite electrodes by equivalent node electrodes with an optimised placement. Alternatively, the electrodes can be very accurately represented by including them as finite bodies in the model, e.g., with the Complete Electrode Model (CEM), as described in Rücker \& Günther (2011). This requires 3D modelling. In our study, we incorporate CEM electrodes in all of our models.

With our ERT borehole-to-surface (abbreviated as "b2s") field setup, we can detect the dimensions, depth and resistivity of very thin soil layers, i.e., down to about $10 \mathrm{~cm}$ thickness, as described in Ochs \& Klitzsch $(2020)$. For synthetic data and well controlled measurements with the b2s setup in a backfilled trench, we achieved a high resolution over the depth of the borehole tool and accurate resistivity values. However, a demonstration of the setup for a field application is still missing, which we provide with this publication. We show the specific challenges arising from the field application and how we mitigate their effects.

Our study site is an agricultural field with a generally sandy and gravelly soil, intersected by a network of old river channels in shallow depths (palaeochannels) filled with clay and silt. It was subject of several other studies especially in the context of the Transregional Collaborative Research Center 32. von Hebel et al. (2014) show the soil variability with Electromagnetic Induction (EMI) measurements on a nearby field in the same area. Former studies also investigated the distribution of palaeochannels with geophysical methods from the surface (von Hebel 
et al., 2018; Rudolph et al., 2015; Brogi et al., 2020) and combined soil classification maps with a crop health assessment (Brogi et al., 2018).

We aim at resolving the substructures of a palaeochannel. For this, we first imaged the lateral boundaries and shape of the channel by measuring a surface ERT profile across it, then chose eight locations along this profile where we measured b2s profiles. The surface ERT profile is 46 $\mathrm{m}$ long, with an electrode spacing of $50 \mathrm{~cm}$, compared to the b2s array, comprising a $5 \mathrm{~m}$ long surface line, with $25 \mathrm{~cm}$ spacing.

Based on the measured field data, we further develop the small-scale b2s ERT setup. First, because of unreliable measured stacking errors, we devise an error model based on the stacking error information. Then we focus on the mitigation of two main installation related effects:

1. Due to the small electrode distances and the overall small-scale nature of the array, i.e., only $5.2 \mathrm{~cm}$ electrode spacing along the borehole tool, the electrodes have a big impact on the measurements. Therefore, the depth of installation of the borehole electrode tool must be known accurately in the inversion model. However, it is not easy to measure the tool depth in the field with the required accuracy, due to small-scale surface roughness, e.g., from a weathered loose soil layer at the surface or from vegetation. Additionally, we investigate the influence of a tilted tool installation.

2. In the field, we electrically coupled the borehole electrodes to the ground by filling the cavities around the tool with a soil mud, i.e., we need to account for the unknown conductive borehole filling in the inversion.

For addressing these points, we conduct a synthetic study, as described in section 3, after introducing the methodology in section 2 .

\section{Methodology}

For simulating and inverting data from the b2s electrode array, we apply the 3D modelling routine depicted in Ochs \& Klitzsch (2020) using the CEM. The geometry of the borehole electrode rod with 20 ring electrodes and the 20 surface electrodes are included as 3D bodies in the forward and in the inversion mesh. This is necessary due to the small electrode spacing, 25 $\mathrm{cm}$ for the surface electrodes and $5.2 \mathrm{~cm}$ for the borehole electrodes (midpoint to midpoint), with electrode lengths of $10 \mathrm{~cm}$ and $4 \mathrm{~cm}$ (tool diameter), respectively. This violates the threshold of 0.2 for the electrode length to spacing ratio for point electrodes, as introduced by Rücker \& Günther (2011) and demonstrated by Ochs \& Klitzsch (2020). The geometric factors are calculated according to the positions, shape, and size of the electrodes, represented via the CEM. 


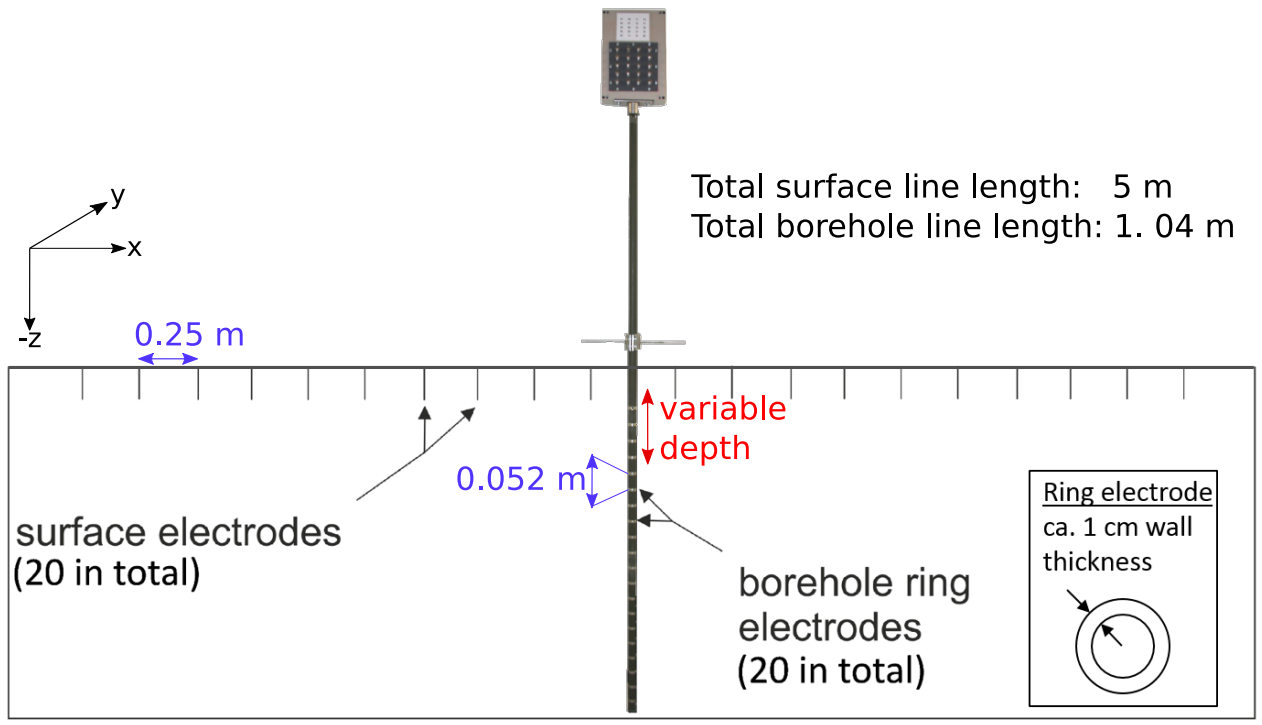

Figure 1: The b2s measurement setup with the borehole tool in the middle, with a diameter of $4 \mathrm{~cm}$ and 20 electrodes, with a total length of $1.04 \mathrm{~m}$. The 20 surface electrodes have a spacing of $25 \mathrm{~cm}$, while the 20 borehole electrodes are spaced by $5.2 \mathrm{~cm}$, resulting in a surface line length of $5 \mathrm{~m}$ and a borehole line length of $1.04 \mathrm{~m}$.

Figure 1 shows the geometry of the electrode array, depicting the surface electrodes as rods and the borehole electrodes as rings. The electrode tool is situated in the middle of the surface array and the surface electrodes have an active length of $10 \mathrm{~cm}$, i.e., they are pushed $10 \mathrm{~cm}$ into the ground. Each of the 20 borehole electrodes is accessible via the console at the top and can be connected to an electrode unit of our measurement device. 
a)

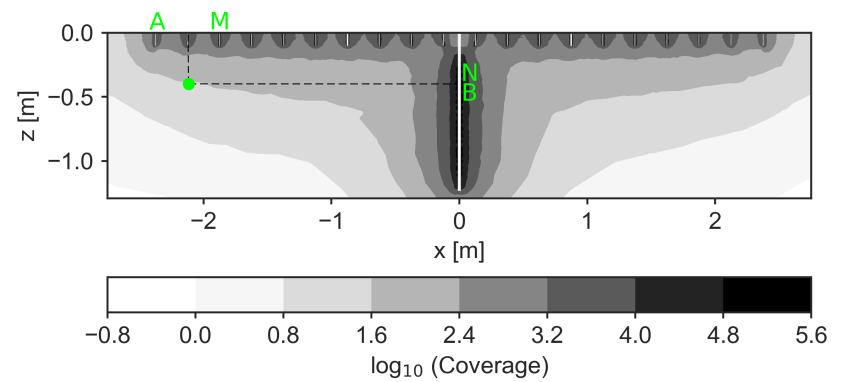

b)
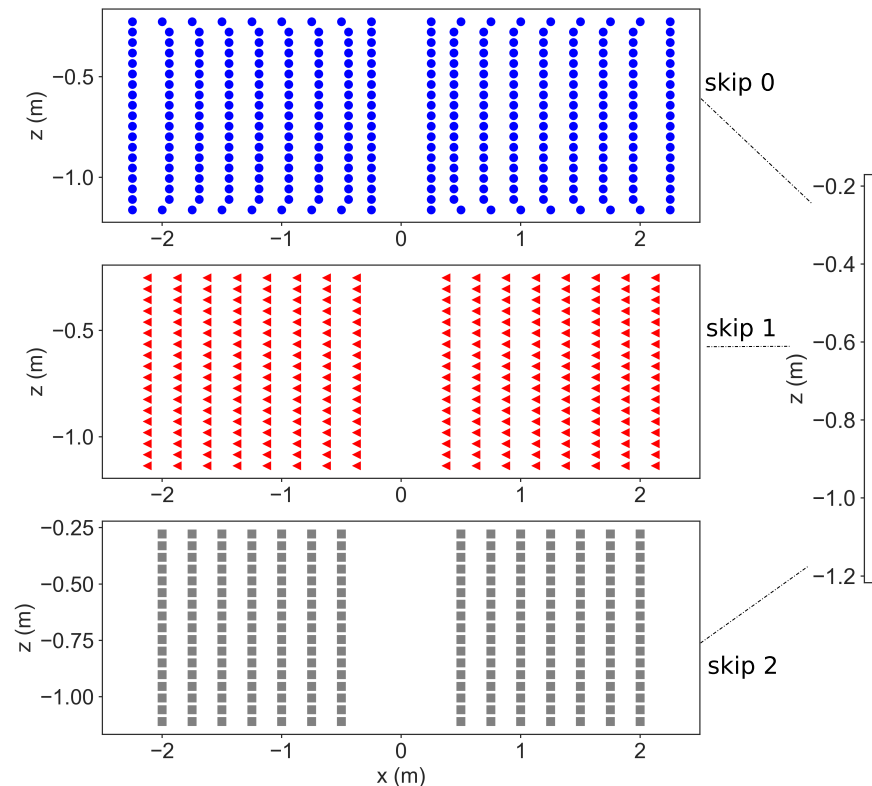

All skips

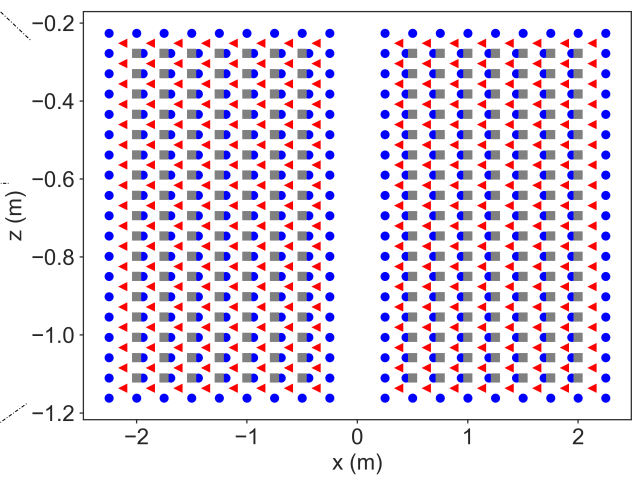

Figure 2: a) 2D slice of the coverage (logarithm of summed absolute sensitivities), and b) pseudosection of the crossed dipole surface-borehole configurations used in this study. The green point in a) marks the position of the data point in the pseudosections for the depicted four-point configuration as an example. The three layers of the pseudosection, defined by the skip size within the crossed dipoles $(0,1$, and 2$)$, and their combination into one pseudosection containing all data are depicted. The zeroth skip data partially overlap with skip 2 . For visibility, the overlapping points are shifted slightly to the right, as shown in the figure. Notice also the different markers and colours used for each skip. 
Due to the geometry of the b2s array in combination with the measured configurations we do not calculate a classical pseudosection for the data, i.e., using the geometric factors for an estimation of pseudo-depth. Instead, we construct the pseudosections based on the midpoints of the dipoles. We demonstrate this for the b2s data in figure 2. In a), we show the coverage, i.e., the sum of absolute sensitivities, of a b2s array. The depicted slice, as well as subsequent inversion results from the b2s array, is an interpolated 2D slice of the 3D model. The 2D mesh is solely used for visualisation of the results (for more details we refer the reader to Ochs \& Klitzsch (2020)).

The coverage develops in a T-shape around the borehole and the surface electrodes for the crossed dipole configuration in use. One quadrupole of electrodes is marked in green, showing the position where the data point is plotted in part b), i.e., at the x- and z-midpoint of the quadrupole. In b), we can clearly distinguish between the three skip sizes used in our list of configurations, i.e., from using neighbouring electrodes to a separation of three electrodes. All three skip classes are combined in the complete pseudosection shown on the right side of figure 2 b). When inverting b2s field data, we need to consider the following aforementioned aspects of the field measurement procedure:

1. The electrode tool position in the subsurface is unknown to a certain degree, and

2. the borehole electrode tool is surrounded by a conductive mud with an unknown resistivity for better electrical coupling to the subsurface.

For the first point, we first have to distinguish between a placement error and the effect of a heterogeneous resistivity distribution. For this investigation, we consider different layered models in a synthetic study and observe the effect of placement errors on the synthetic data and on inversion results. We do not consider the measurement of the borehole tool angle in the field, since it is practically infeasible. However, we can estimate the tool depth in the field by measurement. The second point also requires forward and inverse modelling. The mud was mixed on site with tap water and its resistivity was not measured. Additionally, after filling the cavities around the electrode tool, the mud exchanges water and ions with the surrounding soil, which might change its resistivity over time. We simulate a measurement in a model with a conductive borehole filling around the electrode tool and compare different approaches in the inversion, i.e., decoupling the mud region and finding its single resistivity in the inversion, compared to freely inverting on the mud resistivity without an extra region. In the end, we apply the best solution based on our findings to the field data.

\section{Characterisation of borehole electrode positions and a borehole filling in small-scale b2s ERT}

In our synthetic study, we consider the uncertainty in the placement of the borehole tool in the subsurface. We study both the influence of tilting and of the tool depth, i.e., vertical placement uncertainty. 
a)

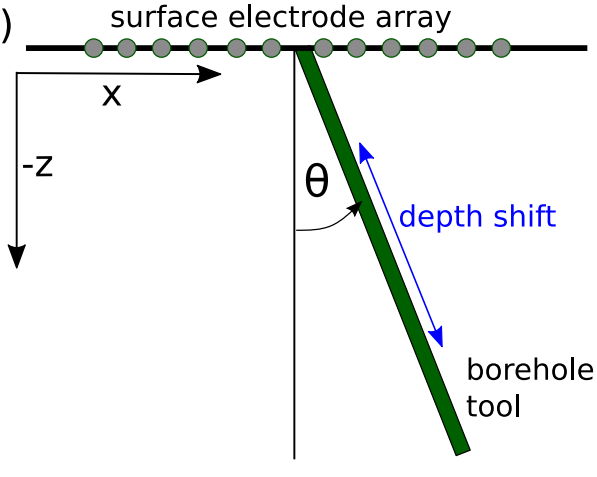

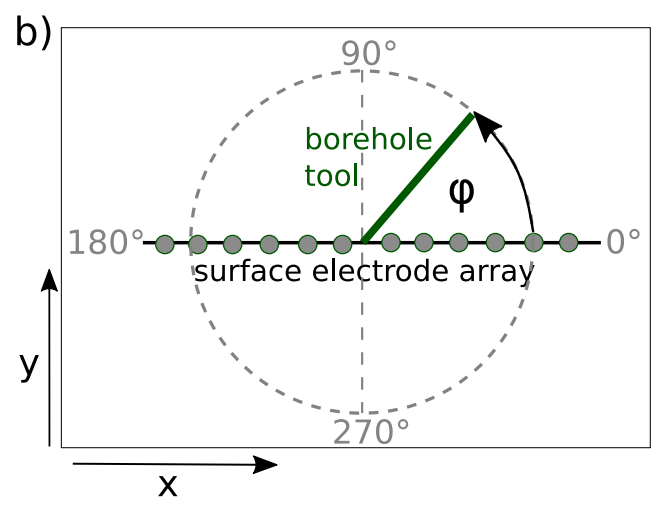

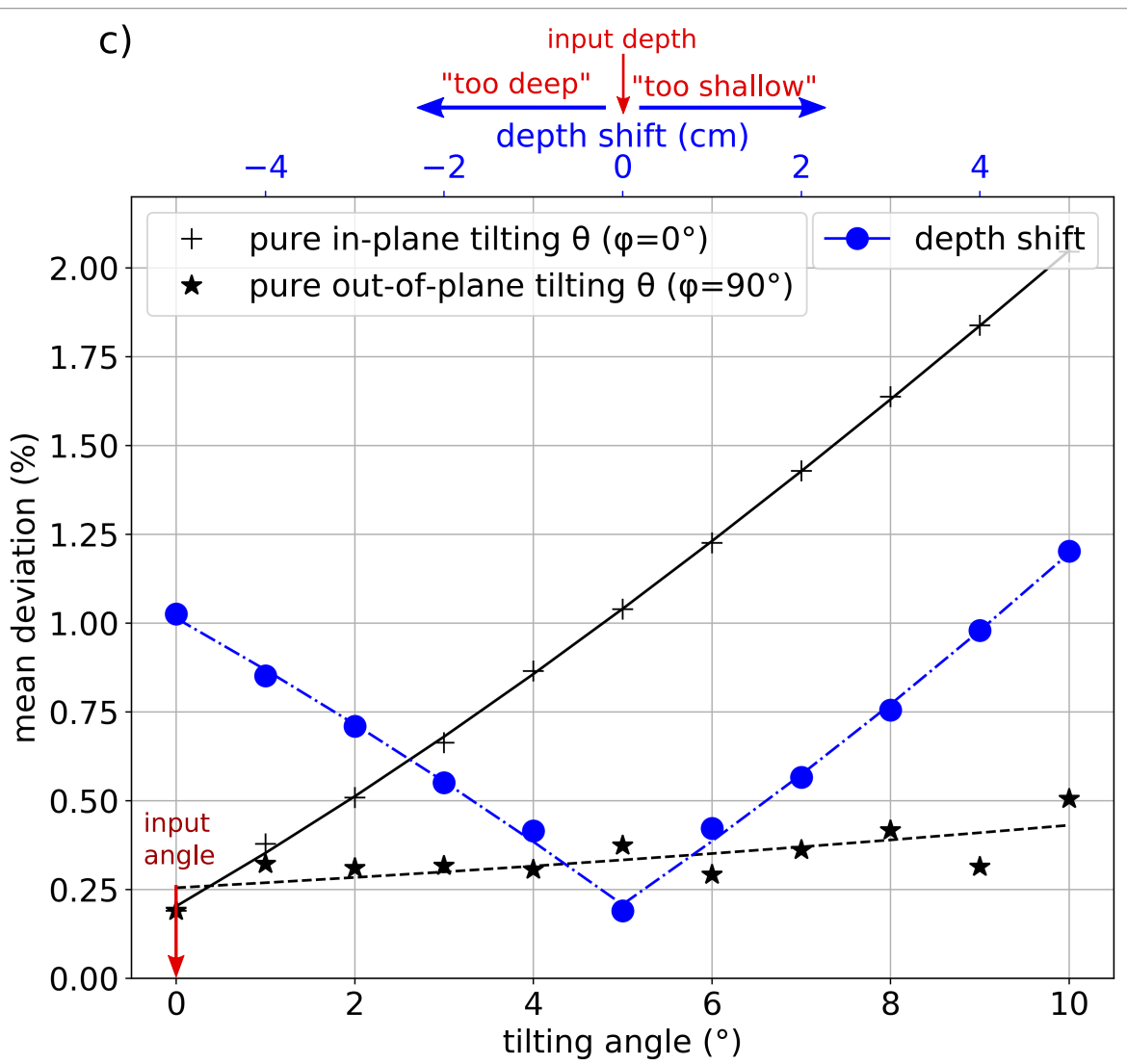

Figure 3: The two angles defining the deviation of the tool from a) the vertical $(\theta)$ and from b) the plane containing the surface electrode array $(\varphi)$, starting with $0^{\circ}$ in positive $\mathrm{x}$-direction and increasing counter-clockwise. c) Influence of tilting or a depth shift on noise-free data. The mean deviation from the homogeneous value of $100 \Omega \mathrm{m}$ is plotted for different input angles and depth shifts. The tilting angle is plotted in black (lower $\mathrm{x}$-axis) and the depth shift in blue (upper $\mathrm{x}$-axis). Both tilting angles are tested for. 
With regard to tilting, we test the sensitivity of the ERT data to two tilt angles, $\theta$ and $\varphi$ (see figure $3 \mathrm{a}$ and $\mathrm{b}$ ). With these angles and the depth of the topmost electrode, which determines the depth of the whole tool, we can fully describe the position of the electrode tool in the subsurface. The depth of the electrode tool must be defined in relation to the positions of the surface electrodes. We refer to a tilting in the x-z plane as in-plane tilting in the following, i.e., within the plane containing the surface electrode spread as well as the borehole tool (a). On the other hand, a tilting in the y-z plane is referred to as out-of-plane tilting (b). We set up a homogeneous model with a vertical tool to generate synthetic resistance measurements. We then calculate apparent resistivities from the simulated resistance values by numerically determining the geometric factors in an additional forward calculation, assuming different $\theta$, from $1^{\circ}$ to $10^{\circ}$. This range is not very large, but we deem it realistic, since in the field a certain effort is taken to ensure a nearly vertical installation. We set $\varphi=0^{\circ}$ and $\varphi=90^{\circ}$, respectively, and compute the in-plane and out-of-plane deviation over the whole range of $\theta$ for both angles of $\varphi$ separately. The tool tilts within the sensitive plane $\left(\varphi=0^{\circ}\right.$ ) from $1^{\circ}$ to $10^{\circ}$, and at $\varphi=90^{\circ}$ (out of the sensitive plane), also from $1^{\circ}$ to $10^{\circ}$. Additionally, we shift the tool in depth by $5 \mathrm{~cm}$ in each direction (a negative shift corresponds to a placement that is too deep and a positive to a placement that is too shallow, compared to the correct placement), also separately. A depth misplacement of 5 $\mathrm{cm}$ corresponds to the borehole electrode spacing, but due to loose topsoil and uneven ground, combined with a tilted tool installation, the depth placement error could become even higher in extreme situations. To a certain degree, this assumption is constrained in the positive direction by the tool electrodes being completely submerged in the ground or sticking out, which can be checked visually. However, even this visual inspection can be misleading, i.e., it can seem that all electrodes are in the subsurface, but loose soil or vegetation around the topmost electrode can change the actual depth reference to a deeper level, with regard to the surface electrodes. Figure 3 c) summarises the effects as a mean overall deviation from the true model value of 100 $\Omega \mathrm{m}$. Due to the numerically calculated geometric factors for the CEM electrodes we have a numerical effect on the calculated apparent resistivities. Therefore, they slightly vary from the true model value of $100 \Omega \mathrm{m}$, even for the correct position of the borehole tool, i.e., we do not reach zero for the mean deviation, although the data are noiseless. A considerable influence on the model comes from a depth shift along the tool axis. On the other hand, the out-of-plane portion of the tilting has almost no influence on the resulting resistivity distribution. However, an in-plane tilting has a recognisable influence on the result and must be addressed.

For point electrodes, i.e., when the electrodes are represented by mesh nodes in the model, we could jointly invert for the subsurface resistivities and the electrode positions as demonstrated by Wagner et al. (2015). However, this approach uses a common model vector for resistivity values and electrode positions, and relies on using the same mesh throughout the optimisation. With CEM, the electrodes cannot be placed freely in a given mesh as nodes but need their correct position and size. They require a new mesh for every shift of the electrode tool. This complicates a direct inversion on electrode positions alongside the resistivity inversion, since the mesh cells, and therefore the length and cell attribution of the model vector, change with every shift of the electrode tool. Instead, we utilise the data vector rather than the model vector for the optimisation and optimise the tool position independently from the inversion. The data vector has the benefit of being independent from the mesh. With this approach, we do not need inversions, avoiding the influence of smoothing and other user input on the result. The model vector also includes the information contained in the data, i.e., the sensitivity pattern and geometric factors, which propagate into the model, but it is superimposed by the inversion process. Therefore, we decided to only show the results for the data. The propagation of errors arising from uncertainty in electrode positioning was shown by Oldenborger et al. (2005) and Zhou \& Dahlin (2003) to lead to significant perturbations in the near-electrode regions in inverted 2D models, sometimes exceeding $20 \%$ in magnitude. 
a) $\theta=1^{\circ}$

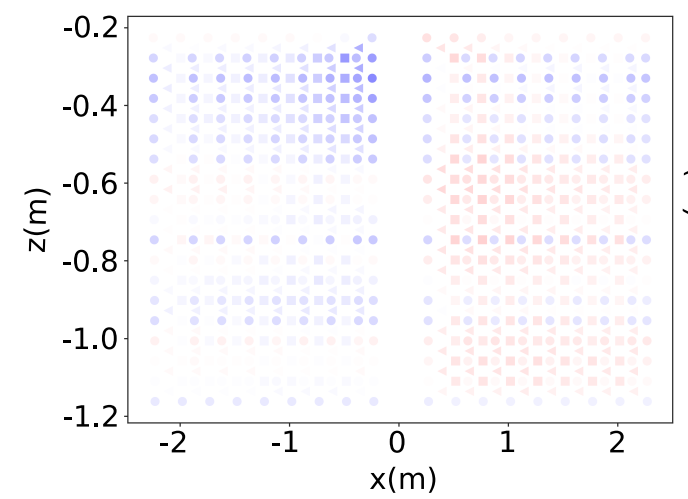

b) $\theta=5^{\circ}$

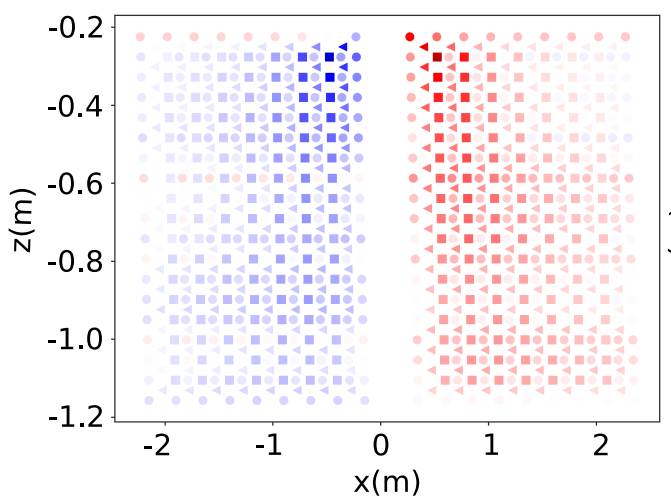

c) $\theta=10^{\circ}$

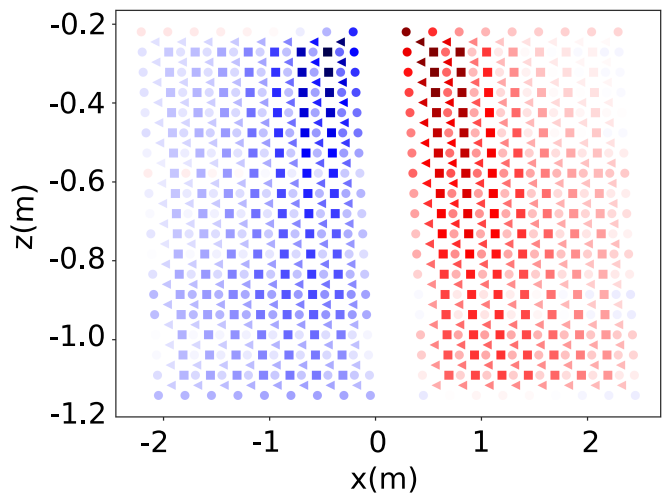

d) $-5 \mathrm{~cm}$ (too deep)

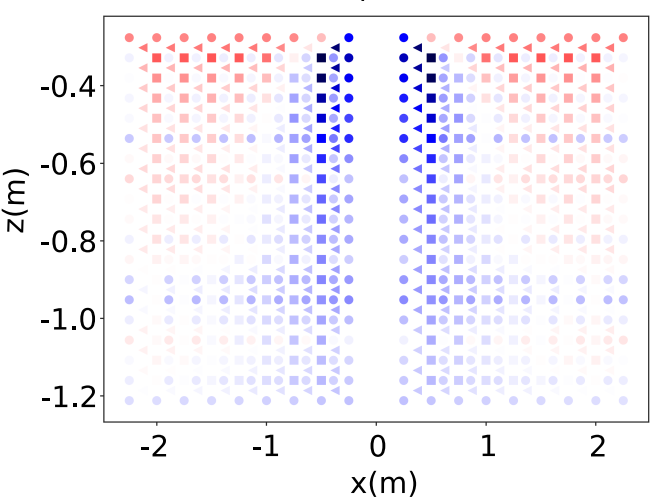

e) $+5 \mathrm{~cm}$ (too shallow)

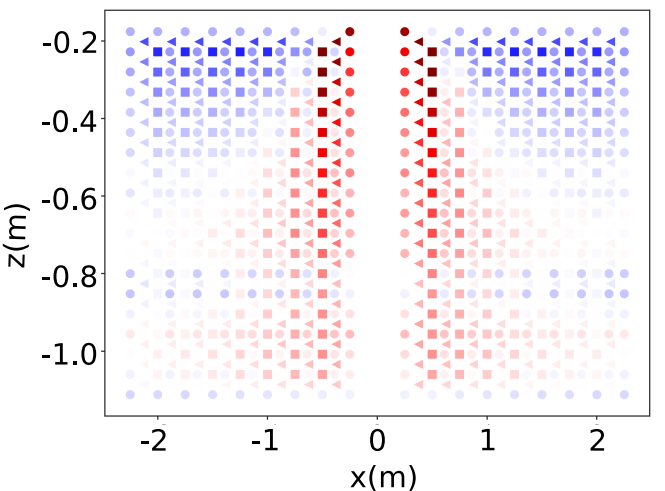

$-10$

Figure 4: The difference to the true model value after calculating new geometric factors for noiseless synthetic resistances from a homogeneous model with $100 \Omega \mathrm{m}$ and a vertical tool: a)-c) Assuming different tilting angles $\theta$ of 1,5 , and $10^{\circ}$, respectively (with $\varphi=0^{\circ}$ ), and d)-e) assuming a depth shift downward and upward by $5 \mathrm{~cm}$, respectively. 
In the field, we measure data with an unknown deviation of the electrode tool from the vertical direction and an uncertain depth. Measured resistances are affected by the position of the borehole electrode tool, due to the current paths influencing the data. The sensitivities of the configurations change as well as the geometric factors, which are used to calculate apparent resistivities. The change in positions of the borehole electrodes caused by a tilting of the tool reflects in the forward data mainly through the geometric factor. Figure 4 shows the deviation to synthetic homogeneous data over a half-space with $100 \Omega \mathrm{m}$ generated with a vertical electrode tool. We see slight deviations from the homogeneous model value in the noiseless data, due to numerical effects caused by working with 3D electrodes, which also propagate into the deviation plots shown here. Small variations emerging close to the electrodes influence the other data points at the same depth. When working with the pseudosections, the viewer needs to keep in mind that the positioning of the measured data points is not necessarily representative of the physical volume influencing certain quadrupoles, but is merely a projection based on the quadrupole midpoints. A more physical representation of current paths would be rather triangular, but harder to review due to overlapping points.

We then compute apparent resistivities from the simulated resistance data on 3D meshes, i.e., we recompute the geometric factors, assuming different angles $\theta$ from $0^{\circ}$ to $10^{\circ}$ in the direction of $\varphi=0^{\circ}$ (a-c). The effect on the geometric factor becomes more pronounced for greater tilting angles. It is very systematic for the surface-borehole configurations used, resulting in a decrease in apparent resistivity on the left side $\left(\varphi=180^{\circ}\right)$ of the borehole electrodes and an increase on the right side $\left(\varphi=0^{\circ}\right)$. We observe the strongest influence at the tool top, close to the surface electrodes, where the coverage is largest, but the effect is visible at the bottom as well. Likewise, a depth misplacement reflects in the data, again strongest at the top where the coverage is high (d-e). If we assume the tool too deep, we see a decrease in apparent resistivity close to the tool and an increase in areas further away from it. If we set the tool too shallow compared to the forward model, we observe the opposite behaviour. From this pattern we conclude that assuming a wrong depth of the electrode tool results in an increase in the standard deviation of the data. 

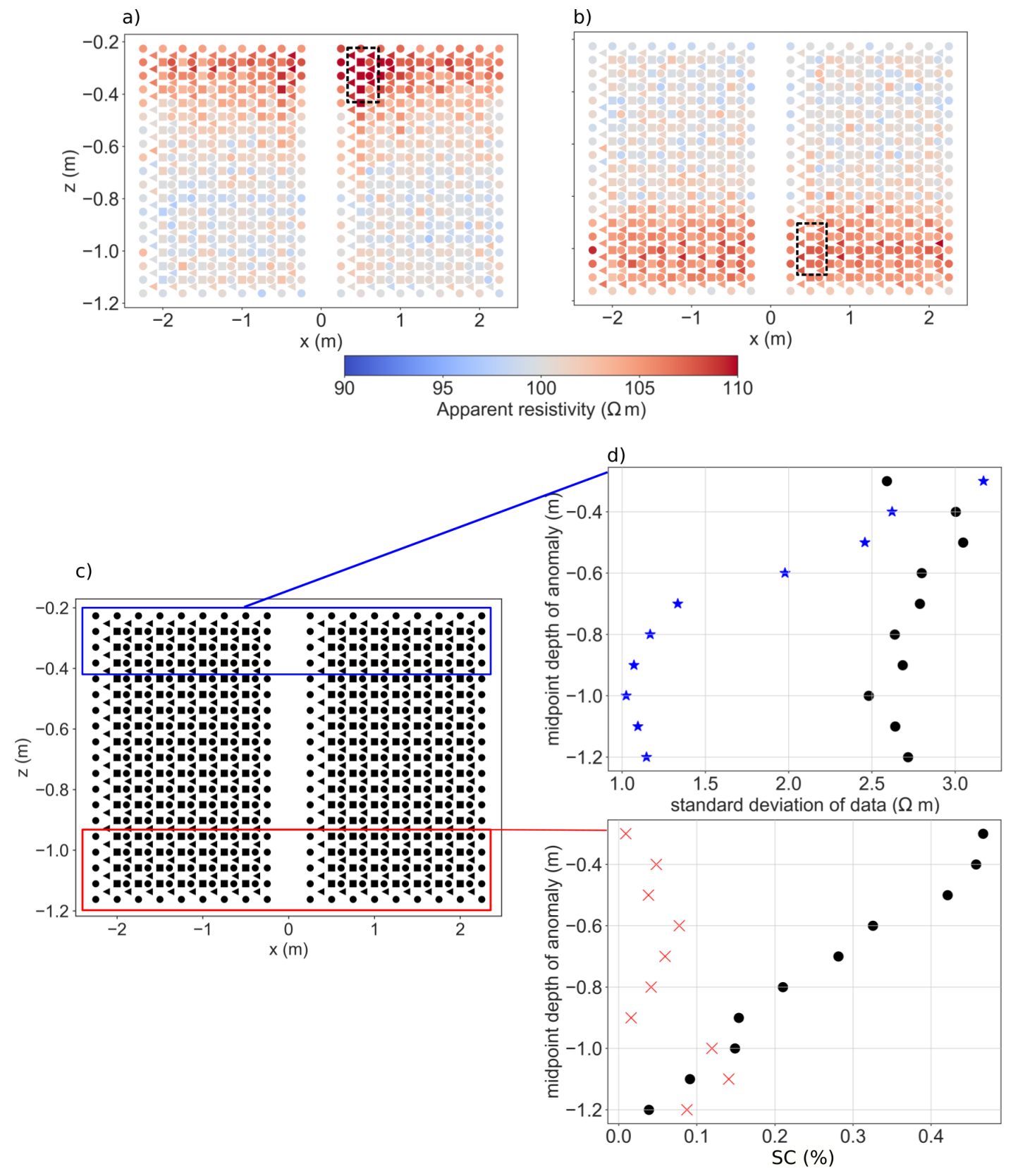

Figure 5: a)-b) Representation of a one-sided anomaly at $30 \mathrm{~cm}$ and $100 \mathrm{~cm}$ depth, respectively, and its influence on the apparent resistivities (the anomaly is represented by the black rectangle with dashed boundaries.) The anomaly has a resistivity of $200 \Omega \mathrm{m}$, in a background of $100 \Omega \mathrm{m}$, and it is infinitely extended perpendicular to the sensitive plane, i.e., in $\varphi=90^{\circ}$ and $\varphi=270^{\circ}$; c) Black points: All data point positions sorted by their quadrupole midpoint. d) Influence of a one-sided anomaly, situated in different depths (shifted in steps of $10 \mathrm{~cm}$ ), on the standard deviation of the apparent resistivities (upper, calculated for determining the depth shift of the tool), and on the symmetry ( $S C$, see equation 1 ) in apparent resistivity between both sides of the borehole tool (lower, used for determining the installation angle of the tool). The standard deviation and symmetry are calculated and plotted for different midpoint depths of the anomaly, considering all data points (black points), and only considering the data points marked in blue (topmost part) or red (bottom part) in c). 
We use the systematic influence of a tilting on the geometric factors to define a criterion for our optimisation on the tilting angle. We calculate the difference between the mean values of apparent resistivities on the left, i.e. $\varphi=180^{\circ}$, and right side, i.e. $\varphi=0^{\circ}$, of the borehole electrodes, as shown in the pseudosections in figure 5 a) and b). A wrongly estimated tilting angle, of the tool within the sensitive plane ( $\mathrm{x}-\mathrm{z}$ plane) results in a systematic difference in apparent resistivity between both sides (see figure 4). This difference is translated into a symmetry criterion, which becomes minimal for the correct angle. However, in reality, a difference between resistivities in the $\varphi=0^{\circ}$ and $\varphi=180^{\circ}$ direction of the tool can also be attributed to asymmetric anomalies. In layered soils, these can be stones or small clay lenses on one side of the electrode tool within the sensitive plane. This effect would overlap with the effect of the tilting, making it impossible to separate both influences. For minimizing the influence of such anomalies on our symmetry criterion, we only use data points at the bottom of the borehole tool. Figure 5 a)-b) demonstrates how for deeper anomalies, i.e., further away from the surface electrodes, the apparent resistivities on both sides become more symmetric. The borehole tool itself has a circular sensitivity, and in the lower model parts the influence of the directional resolution of the surface array becomes smaller. An anomaly on one side of the bottom of the tool is mapped almost equally to the other side, resulting in a more symmetrical image. A tilting of the electrode tool disturbs this symmetry, so we can use it to detect the tilting angle within the sensitive $\mathrm{x}-\mathrm{z}$ plane. Although the coverage is lower at the bottom, we can still detect a difference between both sides, resulting from a tilting of the tool, as shown in figure 4 a-c. For this, we introduce a metric ("SC": symmetry criterion):

$$
S C=a b s\left(\frac{\overline{\rho_{a(\text { left })}}-\overline{\rho_{a(\text { right })}}}{\left(\overline{\rho_{a(\text { left })}}+\overline{\rho_{a(\text { right })}}\right) / 2}\right) \cdot 100[\%]
$$

Our symmetry criterion $S C$ includes a fixed subset of data points, with an equal number of points on the left side and on the right side of the surface electrode array. Figure 5 d) demonstrates how considering all data points (black points) in the calculation of the standard deviation of the data and of $S C$, we see a much stronger influence of the anomaly on the symmetry within the sensitive plane, compared to only considering the data points marked in blue (topmost part) or red (bottom part) marked in figure 5 c). The anomaly is shifted to the discrete depths represented by the plotted points. We calculate the mean value of the apparent resistivities for the chosen 4-point configurations on the left side and the right side, respectively, i.e., the points within the red square in figure $5 \mathrm{c}$ ), and compute the difference (in percent) between the two means, as shown in equation 1 . The calculation of $S C$ allows us to evaluate our data with one simple value per tilting angle of $\theta$, describing the symmetry within the sensitive plane. We recalculate the apparent resistivities from the measured resistances for different tilting scenarios. By comparing $S C$ for many different tilting angles $\theta$ and $\varphi$, we can determine a minimum, which occurs close to the tilting angle that was used as input, in case of synthetic data, or, for field data, to the true installation angle.

The next point to consider is measurement noise. In the calculation of $S C$ for noisy field data, the uncertainty of the mean over $n$ points is decreased by averaging to $p_{\text {mean }}=\frac{p}{\sqrt{n}}$ compared to the noise of $p \%$ of each single measurement point. We include $n=89$ points on each side of the borehole for this calculation. If we assume a noise level of $p=1 \%$, we achieve an accuracy for $S C$ of $p_{\text {mean }}=\frac{1}{\sqrt{89}} \approx 0.1 \%$. This constitutes the limit of resolvability for $S C$, which increases with the overall noise level of the data, since it directly depends on it. For example, if the overall data noise level would be $p=3 \%$, the achievable accuracy of $S C$ would be $p_{\text {mean }}=\frac{3}{\sqrt{89}} \approx 0.3 \%$. 
a) Input angles: $\theta=0^{\circ}, \varphi=0^{\circ}$

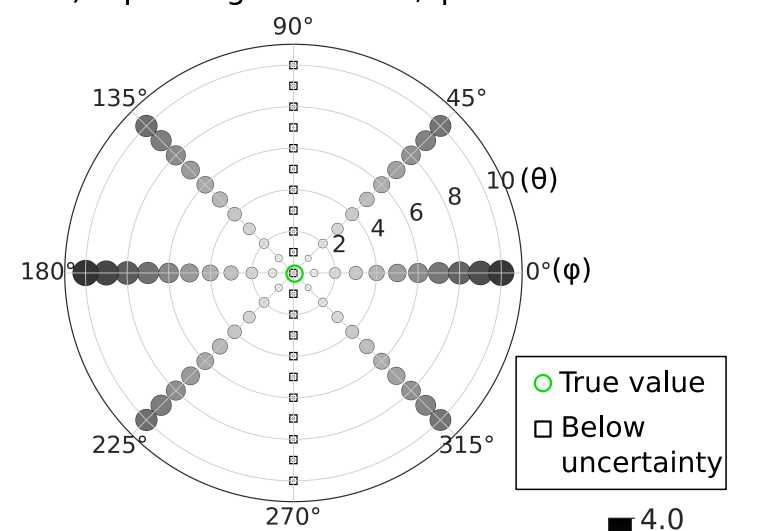

b) Input angles: $\theta=5^{\circ}, \varphi=0^{\circ}$

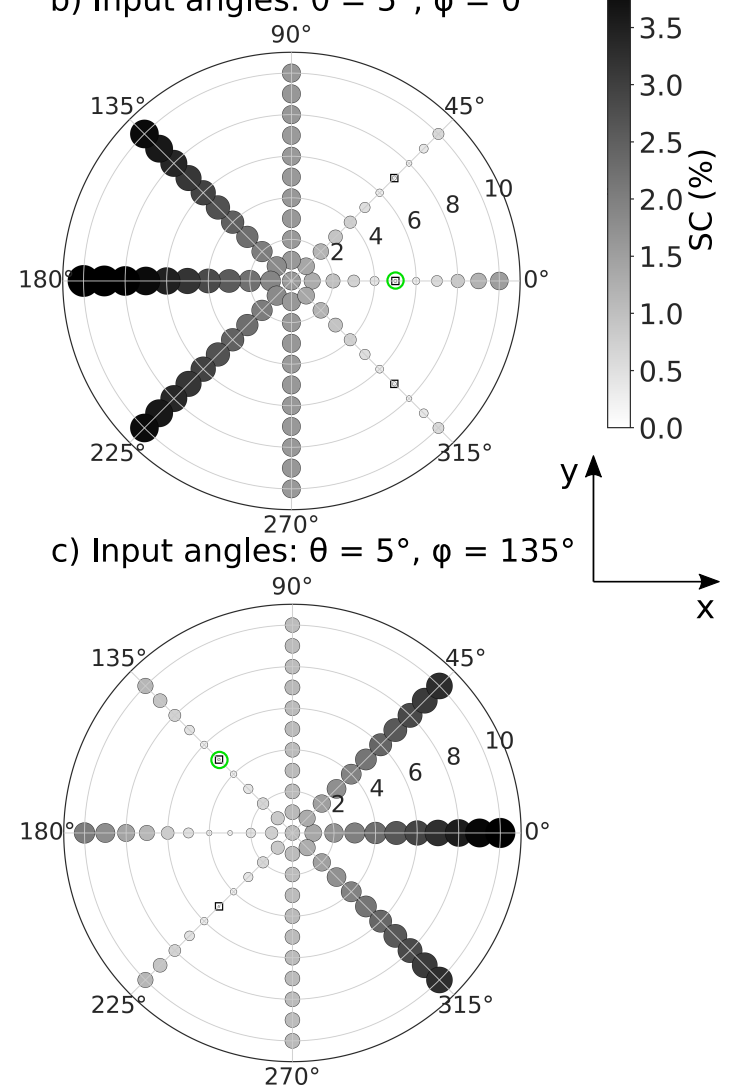

Figure 6: The symmetry criterion $S C$, calculated for different combinations of $\theta$ and $\varphi$ in a homogeneous half space. The circle plots can be read as a topview of the electrode tool, with the tilting angle $\theta$ being plotted as the points spreading out from the centre, i.e., small tilting angles are plotted close to the centre, and larger angles further out. The direction of the tilting is described by the discrete angles of $\varphi$, with $0^{\circ}$ and $180^{\circ}$ denominating a tilting in the sensitive $\mathrm{x}-\mathrm{z}$ plane. The points are scaled and coloured according to the value of $S C$ for the according combination of $\varphi$ and $\theta$. All points with values below the achievable uncertainty of $S C$, based on the applied noise level of $1 \%$, are framed with black squares. The true values, i.e., input angles $\varphi$ and $\theta$, are marked with green circles. 
Figure 6 demonstrates the optimisation process. We have data with an input combination of angles $\varphi$ and $\theta$, describing the tilting of the electrode tool. Then we scan a range of discrete values of $\varphi$ and $\theta$ and calculate our symmetry criterion $S C$ for every tested combination of both angles. Usually we get a couple of comparably small values around the true angle (which is given in the titles of the subplots of figure 6 and marked with green hollow circles ) fitting the original data below the noise dependent uncertainty threshold of $S C$ (black hollow squares). We also already see that the criterion is not very sensitive to the out-of-plane component of the tilting, which is expected. But since an out-of-plane tilting has a negligible influence on the data, we only need to optimise for the in-plane tilting angle $\theta$ and the direction of the tilting (either $\varphi=0^{\circ}$ or $\varphi=180^{\circ}$ ).

We also need a parameter that allows us to find the correct tool depth, since the depth has a big influence on the data and consequently the inverse model. However, the effect of a depth shift on the data is not as easily translatable into a data-based criterion as an angled installation. The effect of tilting can be isolated to a high degree from the resistivity distribution of the subsurface, since a difference between left and right side within the sensitive plane is mainly caused by a wrong angle, if we only consider the points around the tool bottom. A depth shift on the other hand causes a more gradual shift in apparent resistivities and the effect of a pure shift is symmetric about the central axis defined by the tool. Therefore, it is influenced by the subsurface resistivities less predictably than the tilting. A wrong depth creates anomalies at the top of the electrode tool, as can be reviewed in figure 4. An underestimation of the tool depth, i.e., setting the tool too shallow in the model, has a bigger influence on the data than an overestimation, due to the borehole electrodes being closer to the surface electrodes (see figure $4 \mathrm{~d}$ and e). The artefacts introduced by a depth shift lead to an overall increased range of data values especially in the upper part of the model, which is marked by the blue frame in figure $4 \mathrm{~d}$ ). We can use this by looking at the standard deviation. If we only use the points in the topmost part, the effect becomes more distinct and identifiable, compared to using data from all depths. We expect a minimum in the standard deviation of apparent resistivities around the input depth (in case of synthetic data), or the true depth of the topmost electrode (in case of field data). However, in an unknown underground, an increased standard deviation of the data spread can not be exclusively attributed to a depth shift of the borehole tool, but also to an inhomogeneous resistivity distribution. A layered subsurface, as our main application, can produce very similar symmetric patterns in the data, i.e., an increase or decrease of resistivity with depth in regions close to the borehole tool. In contrast, a tilting of the tool can be identified more systematically in a layered subsurface, and even to some extent in the case of small asymmetries, as described above. The optimisation on tilting angle and installation depth comprises two separate steps, i.e., we optimise on the installation angle first and then use a model with the optimised angle as the input for the depth optimisation (as demonstrated in figure 10). Consequently, we optimise the depth along the (possibly tilted) tool axis, not the vertical. This naturally introduces more uncertainty into the depth optimisation, since we already have an uncertainty on the optimised angle, but we observe very distinct minima in $S C$ at the input angle in our synthetic study. Therefore, we do not expect a significant increase in uncertainty in the final result.

\subsection{Optimisation of layered models}


a) Angle optimisation, layered models

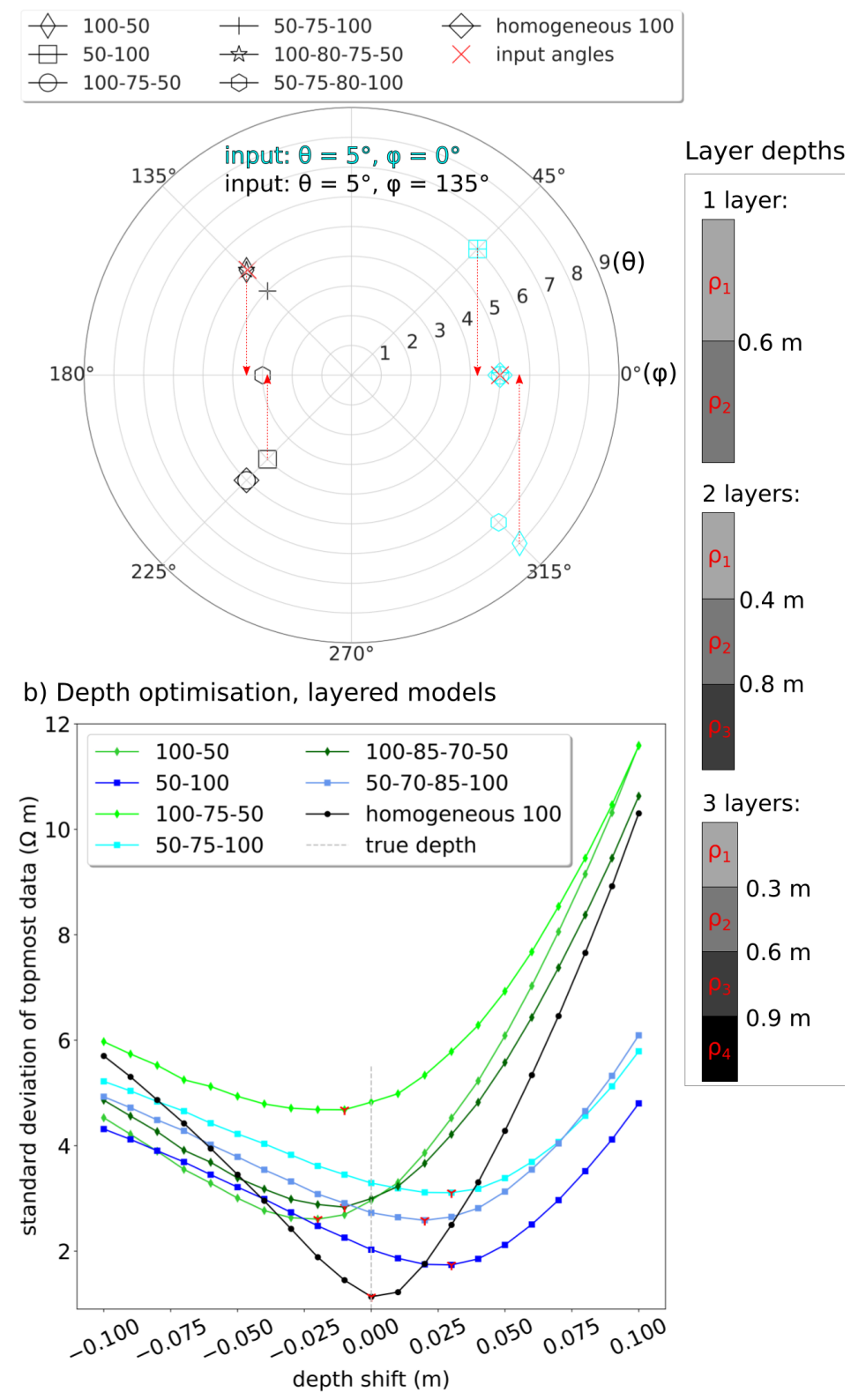

Figure 7: a) The angle optimisation results for different layered models (minima of $S C$ ) The red arrows depict the projection of the combination of $\theta$ and $\varphi$ onto the sensitive plane $\left(\varphi=0^{\circ}\right.$ or $\left.\varphi=180^{\circ}\right)$; b) The depth optimisation results using the standard deviation of the apparent resistivity over the topmost $20 \mathrm{~cm}$ of the pseudosection (blue frame in figure 5 c) for layered models with varying numbers of layers over a homogeneous half space and varying distribution of resistivity. The green shaded lines with diamond markers represent models with decreasing resistivity with depth, while the blue shades with square markers stand for models with increasing resistivity with depth. The black line with circles is the result for a homogeneous model. The grey dashed line marks the true depth, and the red markers the minimum of each curve. 
So far we have only considered a homogeneous model. We mostly expect horizontally layered soils as use cases for our ERT setup. Therefore, we test our criteria for the tilting angle and a depth shift in different scenarios of layered models with various number of layers and increasing or decreasing resistivities with depth, respectively (figure 7). Obviously, we often do not know the exact structure of the investigated soil, but in many natural and agriculturally machined soils predominantly horizontal horizons are observed within the first metre (typically A, B, and C horizon), with changes in the soil characteristics mainly occurring vertically.

We plot the $S C$ criterion as a proxy for the tilting angle in the sensitive plane (figure 7 a). We only show the minimum of each optimisation with the tool position being defined by the respective combination of the two angles $\theta$ and $\varphi$, while the depth of the electrodes is slightly influenced by the angle $\theta$. This implies that stronger tilting reduces the electrode depths, compared to a vertically installed tool. The two different input tilting angles that are tested within each model are marked with red crosses, the corresponding results from the optimisation are colour-coded. We get an estimate of the tilting angle within the sensitive plane, as can be deduced by projection of the resulting combination of angles onto the line defined by $\varphi=0^{\circ}$ and $\varphi=180^{\circ}$. This projection is symbolised by the red arrows in the figure, which also allow an estimate of the maximum variation of the recovered angle $\theta$, which is about one degree between the tested models.

In figure $7 \mathrm{~b}$, we observe the standard deviation of our data in the topmost part (the first 20 $\mathrm{cm}$ ) of the pseudosection as a proxy for a depth shift. The true depth is the input depth for the synthetic model (here depicted as a shift of $0 \mathrm{~m}$ ), assuming a vertical tool without a tilt. The standard deviation is then plotted for the recalculated apparent resistivities for a range of depth shifts. An increasing resistivity with depth (blue shades with square markers in the figure) leads to an underestimation of the tool depth by up to $3 \mathrm{~cm}$, while a decreasing resistivity with depth (green lines with diamond markers) has an opposite, but slightly less strong effect. We can conclude that we are able to resolve the tool depth within a range of 2-3 $\mathrm{cm}$ for the subsurface structures we expect, i.e., layered soils. For this magnitude of uncertainty in depth shift we will already see notable artefacts in our final model. In an effort to reduce this uncertainty, we test another criterion for detecting a wrong tool depth, using the value of the objective function of the inversion result. The complete objective function is defined as follows (Günther et al., 2006):

$$
\Phi=\Phi_{D}+\lambda \cdot \Phi_{M}
$$

It consists of the data functional $\Phi_{D}$ describing the data fit, and the model functional $\Phi_{M}$ which contains the model constraints. The regularisation parameter $\lambda$ influences the smoothness of the resulting model. 


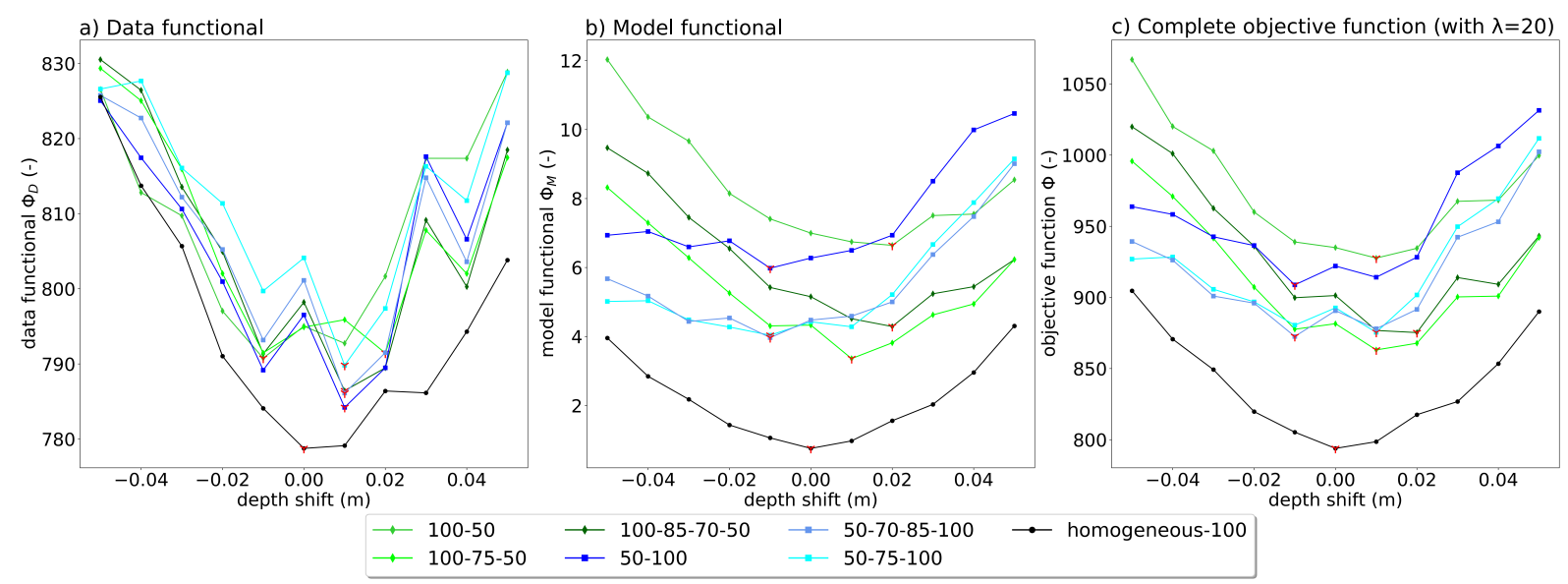

Figure 8: The result of scanning the range of $+-5 \mathrm{~cm}$ tool depth around the input depth. The plotted parameters are a) the data functional $\Phi_{D}$, b) the model functional $\Phi_{M}$, and c) the combined objective function $\Phi$ minimised during the inversion, for a constant regularisation parameter $\lambda=20$. On the $\mathrm{x}$-axis, we plot the depth shift in $\mathrm{m}$, with $0 \mathrm{~m}$ corresponding to the input depth of $-0.2 \mathrm{~m}$.

We set up the same layered models as in figure 7 and invert the synthetic data with different tool depths, as can be reviewed in figure 8 . We keep the regularisation parameter $\lambda$ constant and compare the data functional (a), the model functional (b), and the complete objective function values (c). In figure 8 c) we can see a similar behavior for the different layered models as in figure 7, but the corresponding minimum in the objective function gives an estimation of the tool depth which is closer to the input depth, i.e., for all tested models but one it is within $1 \mathrm{~cm}$ from the true value. The model functional develops in a similar way, whereas the data functional follows the overall final fit, expressed as the parameter $\chi^{2}$ (which indicates whether the model is fitted within the data noise level), very closely. In general, all three parameters are slightly influenced by the inversion parameters and the resistivity contrasts in the subsurface. Nonetheless, we use the objective function as the proxy for the tool depth for our field data, since we found that it gives more accurate results in terms of recovering the input depth than the standard deviation of the data for the tested layered models.

\subsection{Influence of a conductive borehole mud}

In our field experiment, we embedded the borehole tool in a conductive mud for optimal electrical coupling of the electrodes. For this, we augered with a slightly bigger diameter than the tool diameter, lowered the tool down in the middle and filled the surrounding voids with the mud. The mud itself was made from the soil by mixing it with water to make a pourable mud. The hole around the borehole tool was filled from the top and the tool was moved around in the mud to let air bubbles escape. The tool has a diameter of $4 \mathrm{~cm}$, and we estimate a cylinder shell of approximately $0.8-1 \mathrm{~cm}$ around the tool, filled with the mud. If we do not explicitely include this additional conductive material around the tool in our 3D inversion, we get an influence on the inversion result, e.g., different sensitivity patterns due to current channelling in the borehole mud, as demonstrated by Doetsch et al. (2010). The borehole tool itself is modelled as a hollow cylinder. The surface is a no-flow boundary, apart from the electrode surfaces that act as current sources and sinks. 


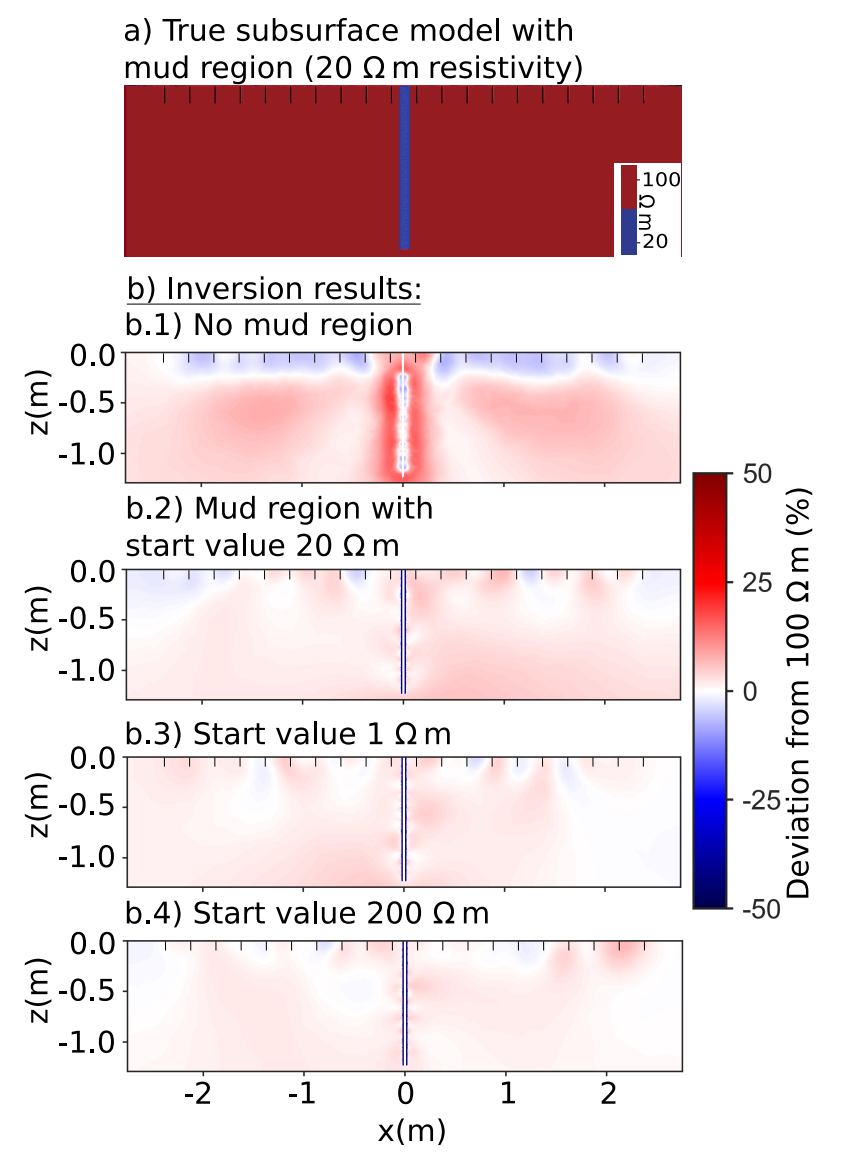

Figure 9: Influence of a mud layer surrounding the electrode tool. a) The input model used for forward modelling the synthetic data with a mud region surrounding the borehole tool; b) Inversion results for the synthetic data for different cases (different inversion meshes): No incorporation of the borehole mud region (b.1), incorporation of the region, assigning the correct (b.2), an underestimated (b.3) and an overestimated (b.4) start resistivity. 
We introduce the borehole as a separate region with a single resistivity value as the starting model. Since we do not know the resistivity of the mud from the field measurements, we provide a first guess for the mud resistivity as a starting point, as well as a lower boundary value of 5 $\Omega \mathrm{m}$ and an upper boundary of $200 \Omega \mathrm{m}$. Then we let the algorithm find the best fitting value during the inversion. For testing the approach, we create synthetic data in a forward calculation with a mud region with a thickness of $1 \mathrm{~cm}$ surrounding the electrode tool all around. We assign it a resistivity of $20 \Omega \mathrm{m}$ and a resistivity of $100 \Omega \mathrm{m}$ to the rest of the model. We invert the data on different inversion meshes applying the following assumptions: 1 . no borehole region in the mesh, 2. borehole region incorporated and with the correct start value of $20 \Omega \mathrm{m}, 3 .-4$. borehole region incorporated with a start value under- and overestimating the true value. As seen in figure 9, we need to account for the borehole mud in a structurally decoupled region in the inversion mesh, allowing for a sharp resistivity contrast. If we invert freely on the borehole mud resistivity without a decoupling, it influences the model outside the borehole too, leading to artefacts (b.1). If we include a distinct region in the inverse mesh and invert on a single resistivity value in this region, the model is negligibly sensitive to the input resistivity for this region. As figure 9 b.3) and b.4) demonstrate, an under- or overestimation of the start value does not affect the final result significantly. 
a) True subsurface model with mud region, tilting angles $\varphi=0^{\circ}, \theta=5^{\circ}$, tool depth $=-0.2$ metres

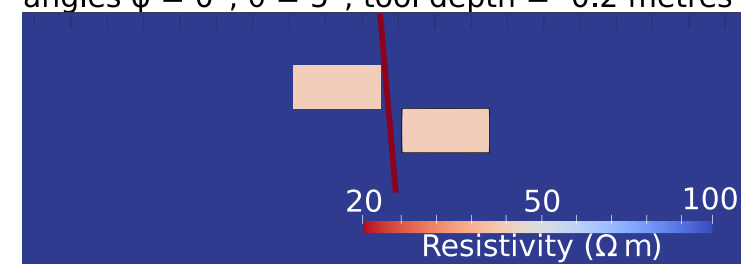

b.1) Angle opt. without mud region c.1) Depth opt. without mud region
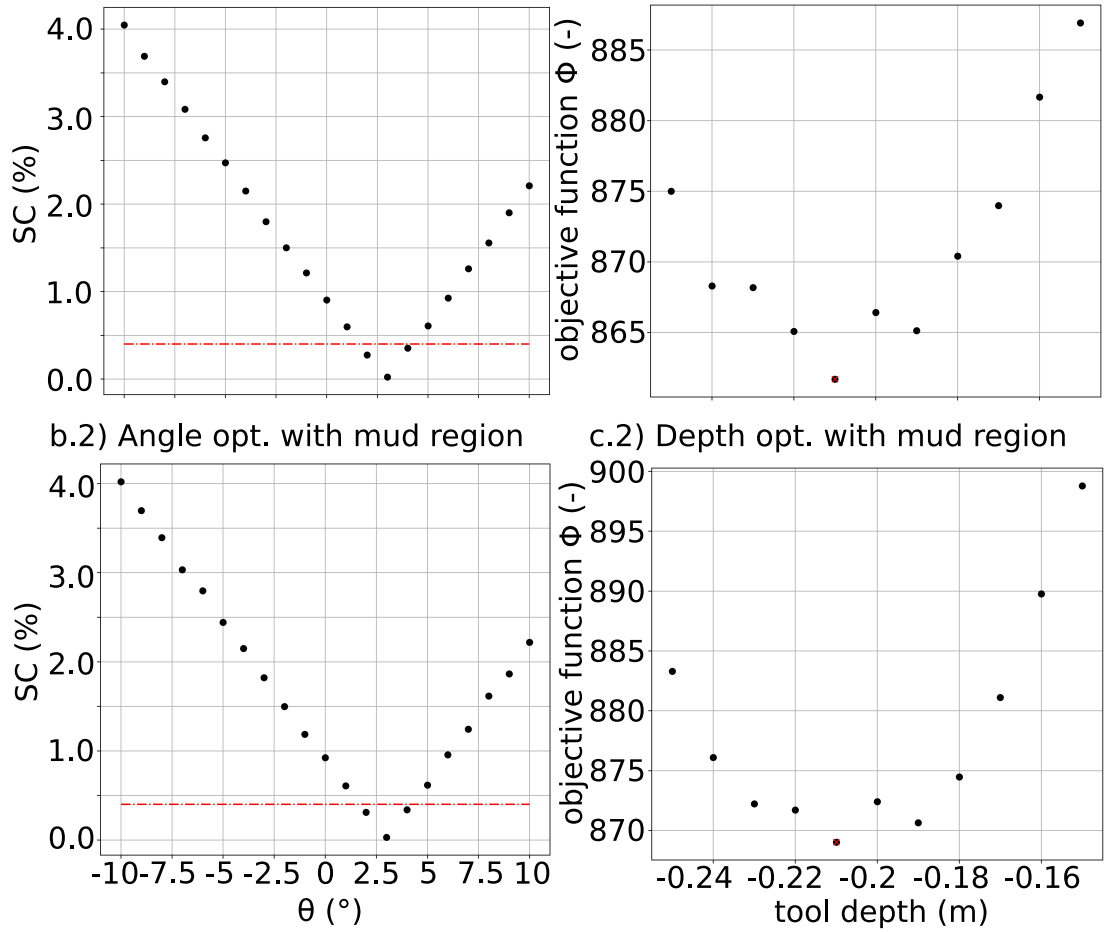

Figure 10: a) Forward model with a mud region and two anomalies. b) Optimisation on borehole tool angle (red line indicating the achievable level of precision) and c) optimisation on borehole tool depth, with the actual depth in m plotted on the x-axis, instead of the shift. The upper row (b.1 and c.1) show the results for ignoring the mud region, while the lower row (b.2 and c.2) show the results for an optimisation with the mud region included in the meshes. 
To test the combined influence of a mud region, a tilting of the tool and anomalies surrounding the tool, we apply our optimisation approach to synthetic data from the model shown in figure 10 a). The half space with a resistivity of $100 \Omega \mathrm{m}$ is disrupted by two anomalies with $50 \Omega \mathrm{m}$ resistivity, on the left and right side of the borehole tool, which is tilted by $\theta=5^{\circ}$ and $\varphi=0^{\circ}$ and surrounded by a mud with $20 \Omega \mathrm{m}$ resistivity. The optimisation results for this model are shown in figure $10 \mathrm{~b}$ ) and c). The angle optimisation recovers the correct direction of the tilting, but underestimates the tilting angle by about $2^{\circ}$, although the two angles surrounding the minimum are also below the maximum achievable precision level, accounting for measurement noise. The relatively big anomalies clearly influence the angle reconstruction. The depth optimisation results in a minimum at $21 \mathrm{~cm}$ depth, which is one $\mathrm{cm}$ too deep. While we see an influence of the resistivity distribution on our two-step optimisation, we do not see an influence of the inclusion of the mud region during the optimisation. We can choose to include the mud region in the mesh which is used to calculate the geometric factors as well as in the inversion mesh, but it does not significantly change the result of the optimisation. Therefore, we will not include the mud region in the meshes for the optimisation on the position of the electrode tool in the field, since it increases the number of mesh cells. The mud region will only be included in the final inversion results. Next, we analyse our field data and show the results of our optimisation and the inversion.

\section{Field application}

We invert field data acquired on an agricultural field situated in the Rur catchment near Selhausen in Germany. It lies in the region of the eastern upper terrace of the ancient Rur river (Weihermüller et al. 2007). The near-surface geology is characterised by Pleistocene sand and gravel (Brogi et al., 2020). This landscape was disrupted by channels formed by melting water after Weichselian glaciation, depositing finer material in this channel system (as described in von Hebel et al. (2018) and references therein). 

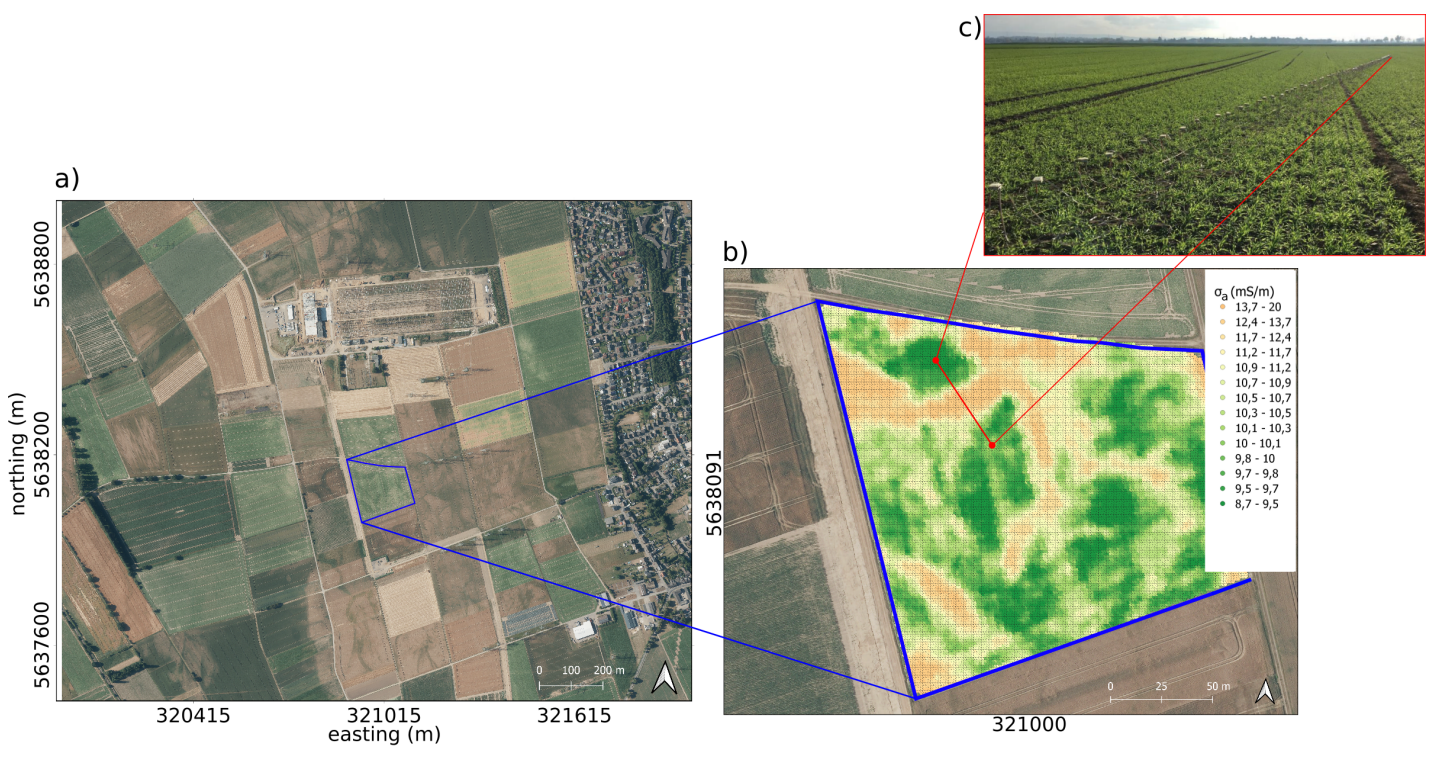

Figure 11: The data acquisition site in Western Germany close to Selhausen.a) The agriculturally used area is characterised by a system of palaeochannels that influences the crop performance and is visible as a pattern on satellite images .b) We show a depth slice at about $75 \mathrm{~cm}$ depth of apparent conductivity from electromagnetic induction measurements, measured on a regular grid on the field considered in this publication superimposed on the satellite image (blue boundary, EMI data are taken from Rudolph et al. (2015) ). Our ERT profile is marked in red and a picture from the field shows the surface electrode array (c).

The crop production on the field is influenced by the subsurface distribution of the palaeoriver channels, beginning at a depth of approximately $50 \mathrm{~cm}$. On top of the channels the plants grow well, while outside of the channel areas the plants experience increased and accelerated wilting during dry periods (von Hebel et al., 2018). The lateral positions of the channels are approximately known from EMI data and aerial photos, and the vertical onset of the channels was further investigated by EMI and ERT (Rudolph et al., 2015$)$. The EMI data shown in figure 11 are also published in Rudolph et al. (2015). Our ERT data comprise a long surface ERT profile perpendicularly crossing one of the palaeochannels (see right side of figure 11, red line) as well as eight b2s measurements along this profile.

\subsection{Field data}

In fall 2017, we initially measured a $46 \mathrm{~m}$ long ERT surface profile over one of the palaeoriver channels with an electrode spacing of $50 \mathrm{~cm}$ as a combined Schlumberger sounding and profiling with 93 electrodes. We then chose several spots along the profile based on the surface data, where we measured with the small-scale borehole-surface array, with a surface electrode spacing of $25 \mathrm{~cm}$. Our goal is an accurate, highly resolved image of the channel geometry and its sub-structures, i.e., thin layering. We concentrated our measurements around the edges and the center of the channel. For the measurements, we drilled boreholes for the electrode rod and coupled the borehole electrodes to the subsurface by inserting a conductive mud, whose resistivity was not measured in the field. For the first b2s measurement there was a problem with the topmost borehole electrode, which was then removed from the measurement scheme. Unfortunately, the same reduced measurement scheme was then used for all subsequent measurements of b2s data. Therefore, we only used 19 instead of 20 borehole electrodes in each 
b2s measurement. We noted the depth of the borehole tool in the field for each measurement location. However, the exact depth of the borehole tool in reference to the surface electrodes depends on the small-scale topography of the soil at the site, as well as on the state of the topmost ploughing layer, which is often loose and not compacted. Therefore, the measured tool depth in the field notes must be considered with caution. Although there was a certain effort taken to drill vertical holes, we did not measure the tool tilt. We conclude that the measured depths are probably not always accurate down to the $\mathrm{cm}$, and that the tilting angles of the tool are unknown. Since this can be a common situation, especially for older data, we consider this a valuable use case for our optimisation procedure.
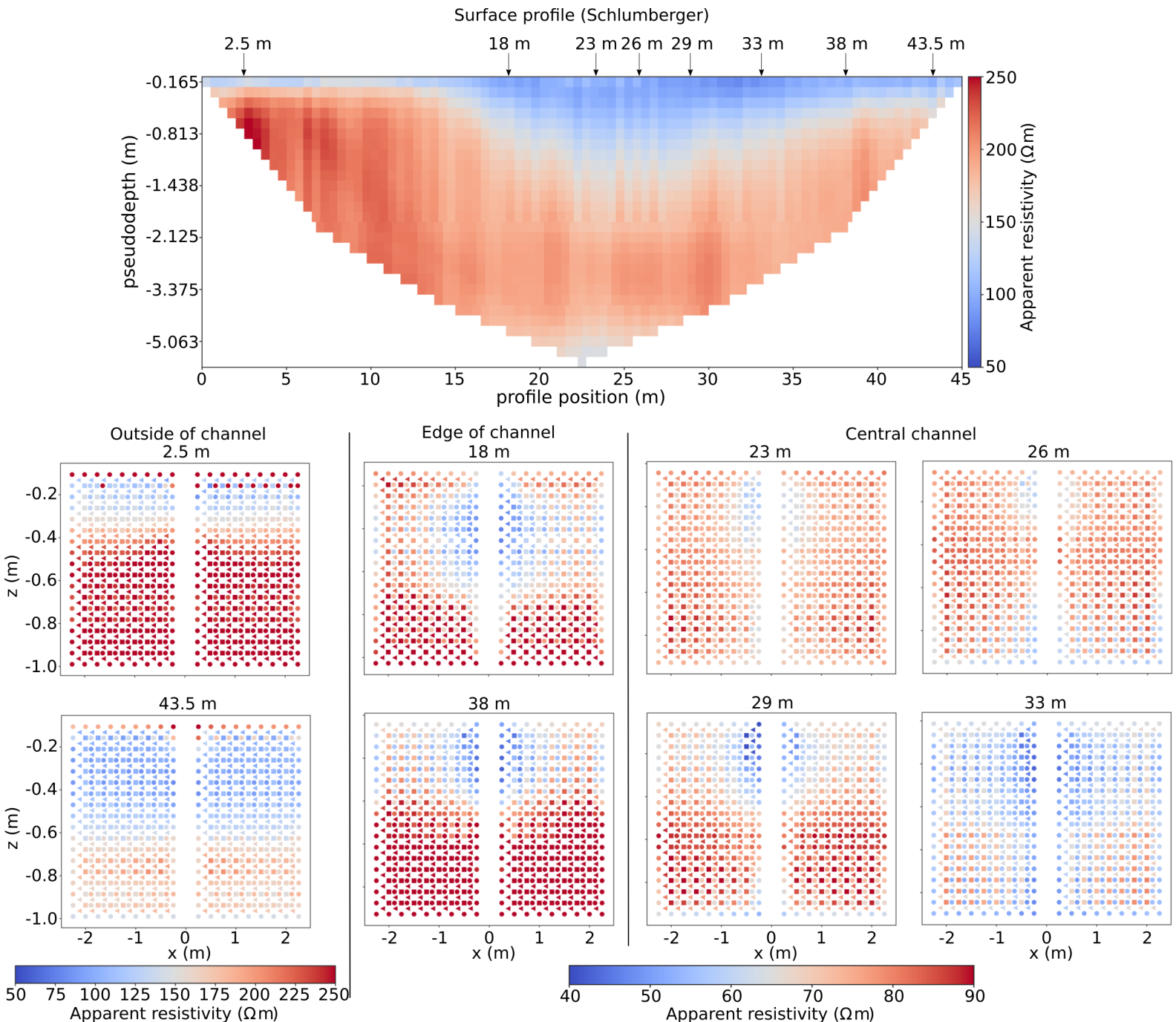

Figure 12: Apparent resistivity pseudosections for the field data. The surface profile is $46 \mathrm{~m}$ long, the positions of the detailed borehole-surface measurements are marked with black arrows. Note the different colour scales due to the overall narrower range of apparent resistivities measured over the channel.

We present the measured data in figure 12 with the tool depth as recorded in the field. The b2s data are measured with a crossed dipole measurement scheme, as described in Ochs \& Klitzsch (2020). It has 820 data points for 19 borehole electrodes and 20 surface electrodes, with the A-M dipole always at the surface and the B-N dipole in the borehole. We increase the electrode skip within the crossed dipoles from zero to two, i.e., going from neighbouring electrodes to a separation of three electrodes (compare figure 2). 

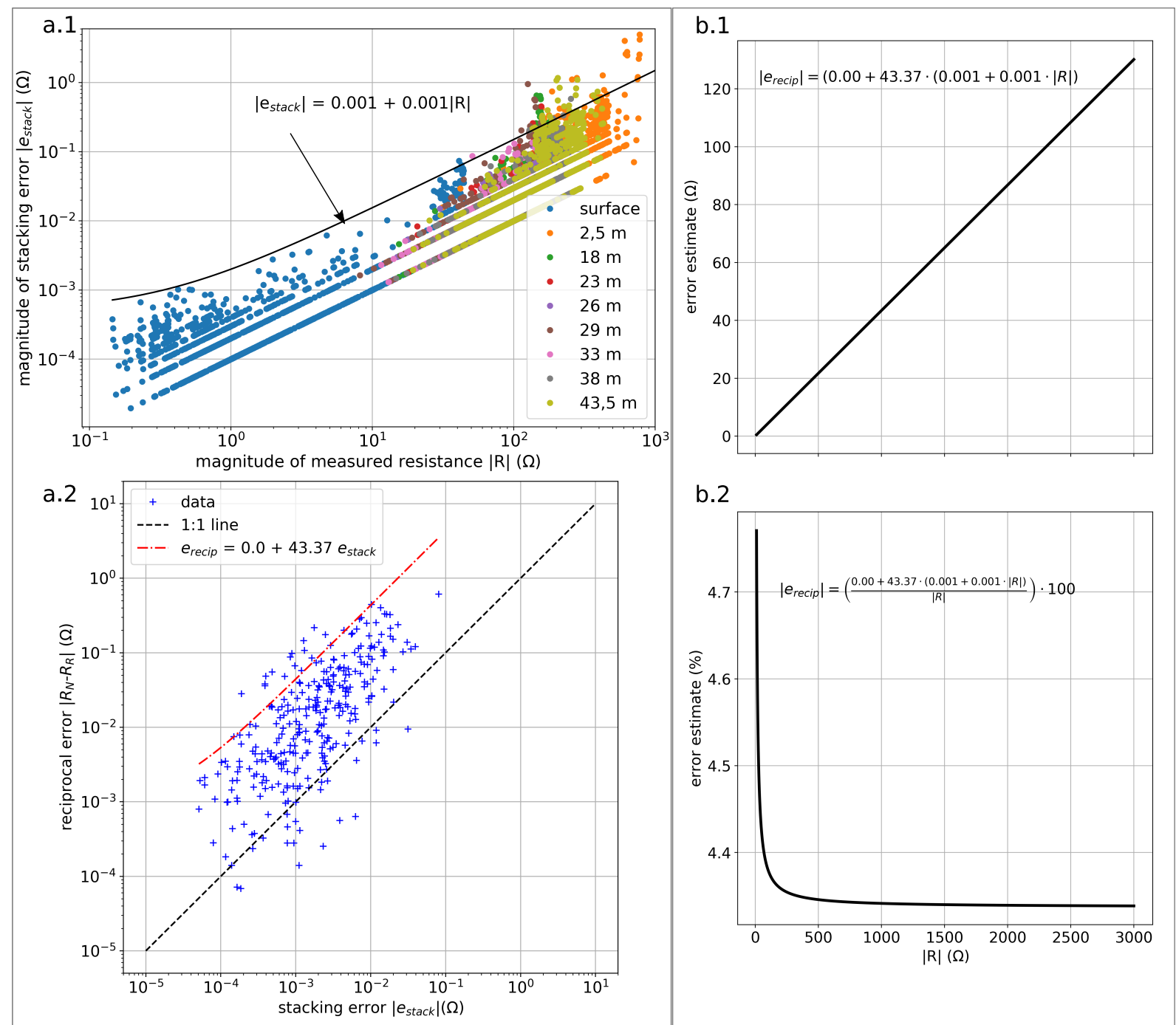

Figure 13: a.1: Double-logarithmic plot of the measured stacking error vs. measured resistance data. The line includes all data without outliers and represents an estimation of the maximum error increase with resistance; a.2: Double-logarithmic cross plot of reciprocal errors and stacking errors for three combined normal-reciprocal dipole-dipole data sets measured at a different site with the borehole tool and the same measurement device. The red line (linear fit encompassing the bulk of the data underneath without some outliers) represents the maximum reciprocal error as function of the stacking error; b: Combined error model (absolute (1) and percentage (2) error) depending on the resistance data. The stacking error model is scaled with the relationship of reciprocal and stacking error. It gives the upper limit of data errors.

Estimating the noise level of field data accurately is important for a correct data weighting during the inversion. For our field data, we only have a stacking error information measured by the field instrument. The stacking error cannot account for systematic errors in a fourpoint array, e.g., badly coupled single electrodes, but rather gives an estimate of the precision of single measurements, which is usually very high (LaBrecque \& Yang, 2001). We did not measure reciprocal data configurations, which would allow the calculation of reciprocal errors. Those rely on one reciprocal measurement by swapping the current and potential electrodes in an independent measurement, whereas we base our error estimation on several repeated current injections and transfer resistance measurements with the same quadrupole. This procedure was chosen because of our single-channel ERT field device, which is the Lippmann 4-Point Light (by 
Lippmann Geophysikalische Messgeräte, Germany). We did not measure additional reciprocal data to reduce the time needed for the measurements. Our standard measurement configuration set comprises 820 data points for the b2s measurements and more than 1500 data points for the surface measurement presented. Instead, we work with the stacking errors as an indicator of data quality. We observe generally very low errors, typically with a few outliers of higher percentage. Over $95 \%$ of the measured stacking errors are smaller than $0.1 \%$, which does not reflect the true data noise and leads to convergence problems in the inversion. This low error estimation is typical for a statistical error based on stacking (Tso et al., 2017; Parsekian et al., 2017). For unknown data errors, the pyGIMLi package (Rücker et al., 2017) has the option to estimate the data errors, following the error model by Friedel (2003). However, the user still needs to give a base error level and a voltage dependent error as an input. We use the characteristics of the stacking error, i.e., the resistance-dependent trend we observe (13 a.1), as a basis for deriving our own error model.

We plot the stacking error against the corresponding resistances and fit a linear model to the observed trend, which is tweaked in order to encompass the bulk of the data, while excluding some outliers (figure 13 a.1). This linear model represents the stacking error, characterized by a very low noise level. For arriving at a more realistic estimate of the overall noise level and the resistance-dependent noise, we incorporate reciprocal error information from additional data measured with the borehole tool at a close-by field site. We combine several data sets from this site, which are dipole-dipole data (normal and reciprocal), measured only with the 20 borehole tool electrodes and the same measurement device as our b2s data. Consequently, we assume the same relationship between stacking and reciprocal error. We plot absolute reciprocal versus stacking errors and fit a linear trend to the data, similar to before, which encompasses the bulk of the data points (red dashed line in figure 13 a.2). Our final error model is a scaled version of the stacking error model, assigning assumed reciprocal errors to the measured resistance (figure 13 b). It represents the upper limit of the error level, depending on resistance: $\left|e_{\text {recip }}\right|=\frac{4.34 \cdot(1+|R|)}{|R|}$ [in \%]. 


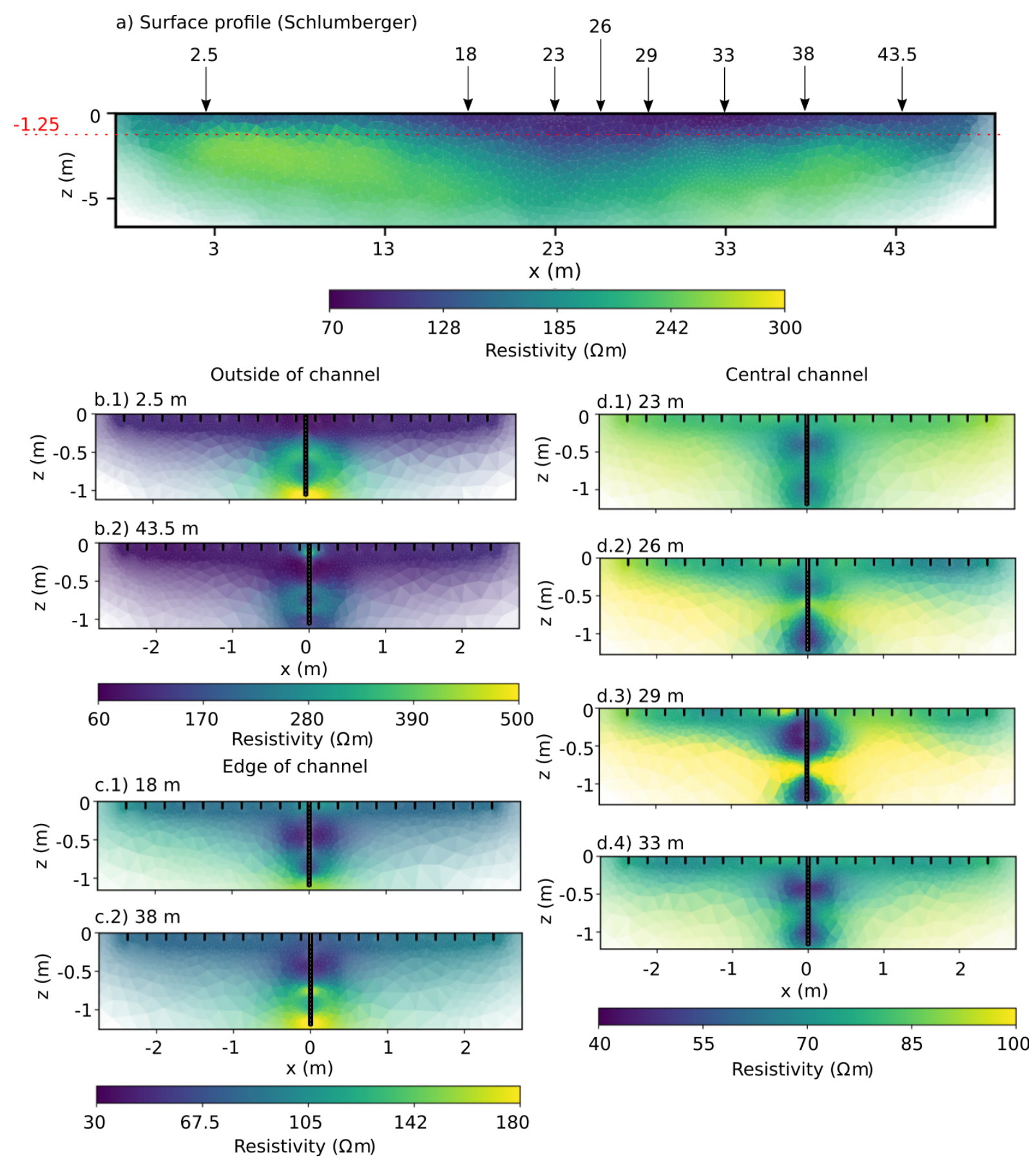

Figure 14: Inversion results of the field data sets. The inversion of the b2s data is done with a mud region included in the mesh and with a vertical tool with the tool depth as noted in the field. The models are shaded with the coverage. a) Surface data inversion, b) b2s results outside of the channel, c) b2s results over the edges, and d) over the centre of the channel.

Before optimising on the tool electrode position, we conduct a first inversion of the field data with the new error model, a vertical tool, and the depth information from the field notes (see table 1). We include the borehole mud region in the inversion mesh and limit the possible resistivity for the mud between $5 \Omega \mathrm{m}$ and $200 \Omega \mathrm{m}$, since this range is realistic for the humid soil we filled in. The results are shown in figure 14 . The surface data, as the b2s data, are inverted on a 3D mesh with 3D CEM electrodes. We see a lot more detail in the b2s models (b-d) than in the surface result (a), both outside and over the channel structure. Next, we 
optimise the borehole tool positions for the b2s field data.

\subsection{Optimisation on borehole tool position}

We search for the projected in-plane tilting angle, i.e., either at $\varphi=0^{\circ}$ or at $\varphi=180^{\circ}$, and the correct depth placement of the topmost electrode for all b2s field data sets. The parameter space is bounded by a tilting angle $\theta$ of up to $10^{\circ}$ and a depth shift from about minus $10 \mathrm{~cm}$ to plus $10 \mathrm{~cm}$ around the depth from the field notes. In some cases, the minimum lies outside of this range, so we extend the search space there. We can recover the correct tool angle and depth placement without considering the mud region around the tool, as can be reviewed in figure 10 . 

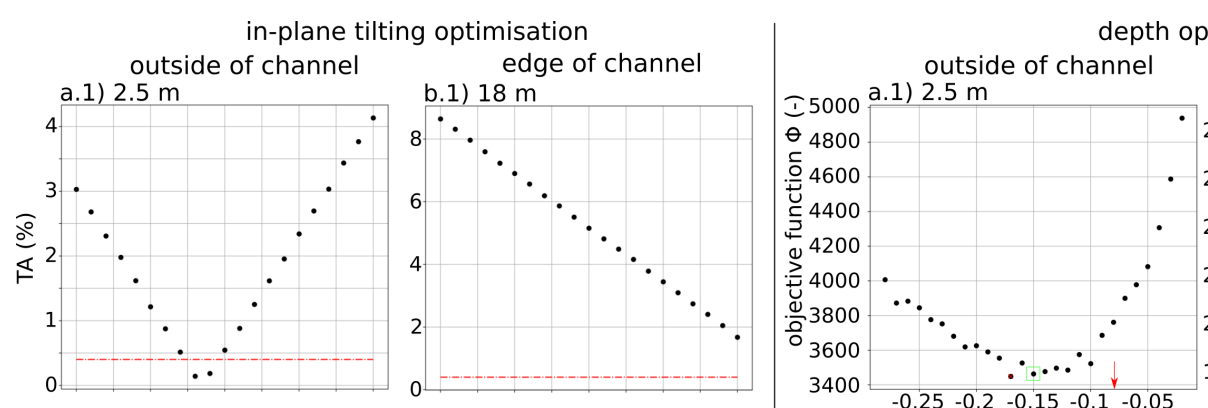

depth optimisation
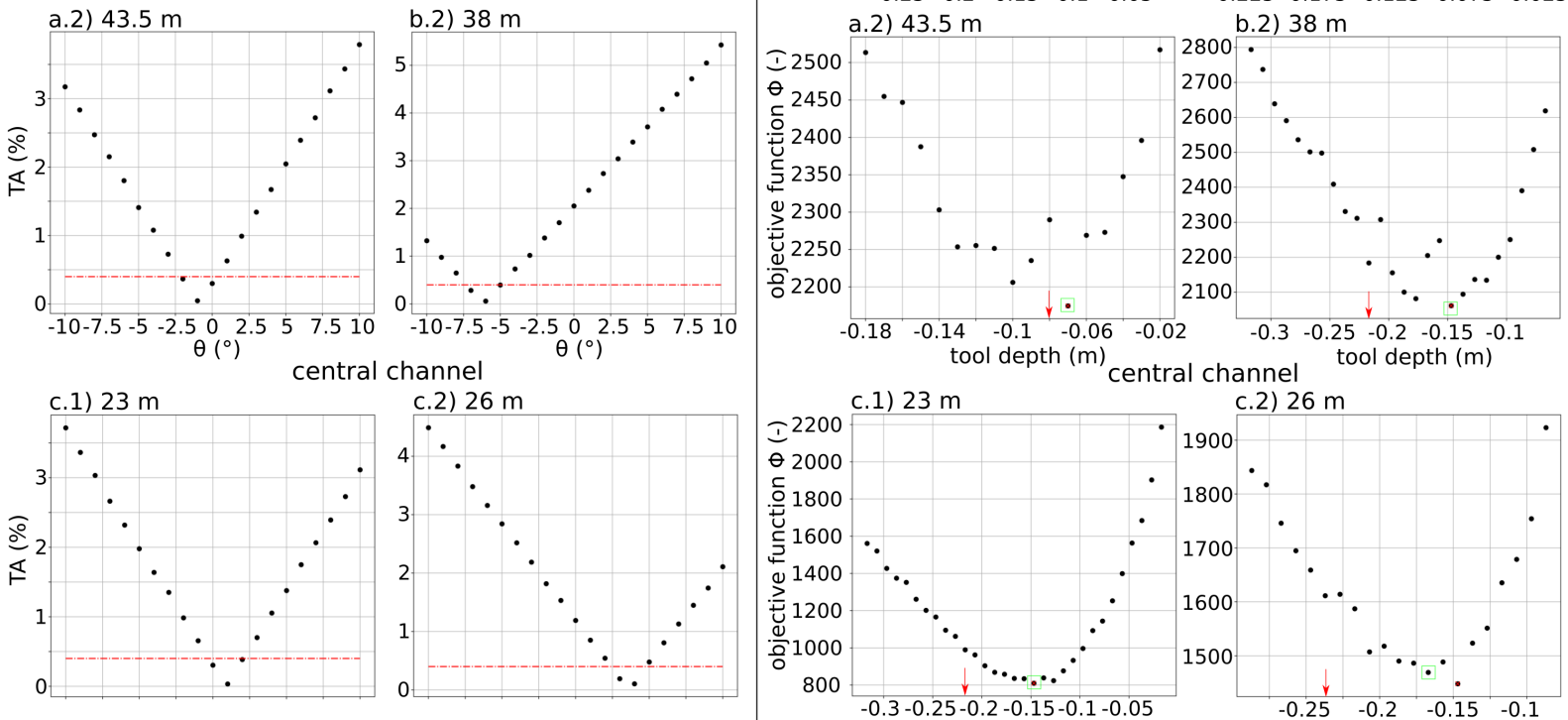

c.2) $26 \mathrm{~m}$
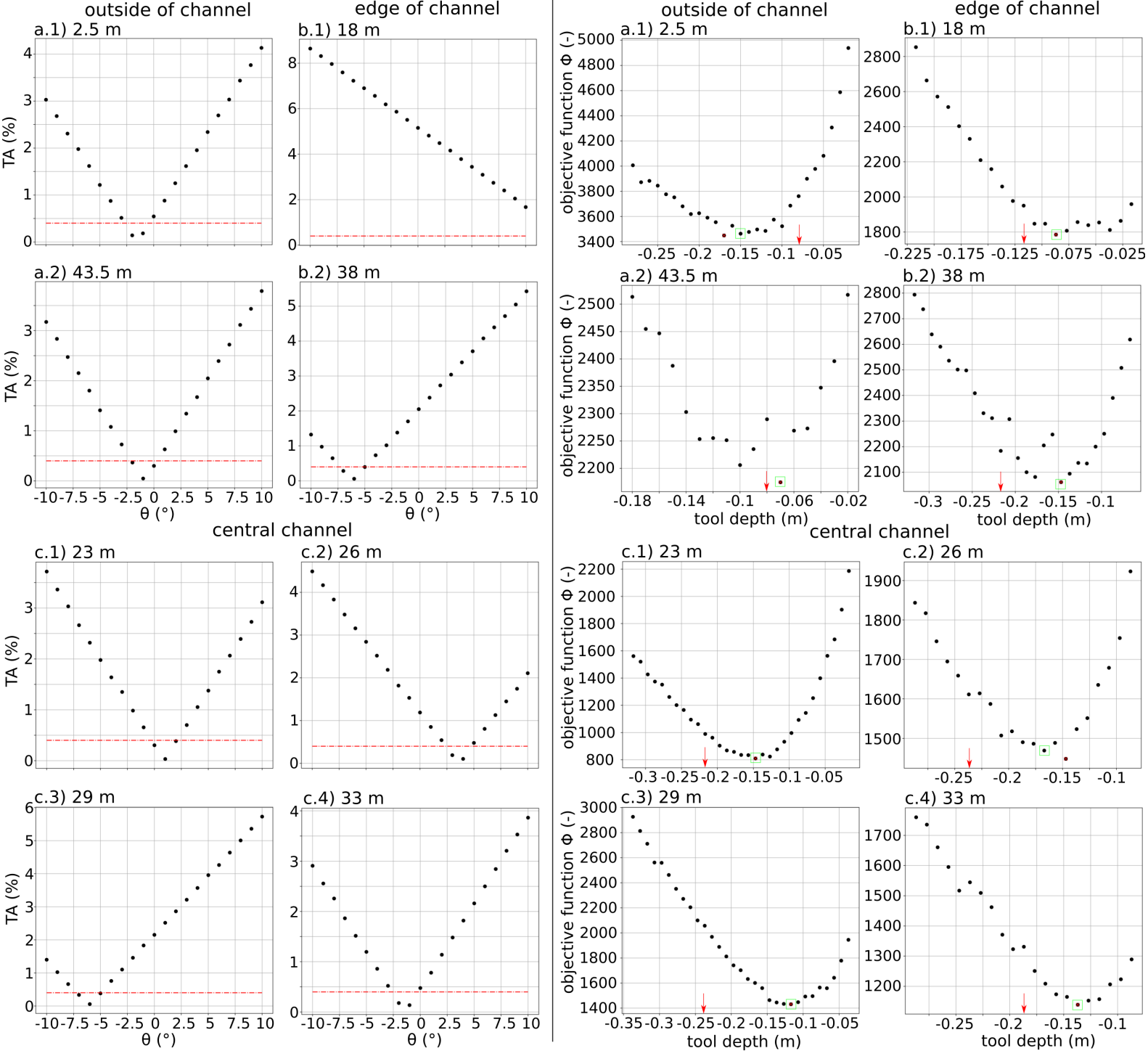

b.2) $38 \mathrm{~m}$

Figure 15: Left: a: In-plane angle optimisation result of data measured outside of the channel, b: measured at the edges of the channel, c: over the center of the channel. The achievable accuracy of $\sim 0.4 \%$ for a base error level of $4 \%$ is marked as a red dashed line. Right: a: Depth optimisation result of data measured outside of the channel, b: measured at the edges of the channel, c: over the center of the channel. The depths noted in the field are marked with red arrows, while the depths used for the final inversion are marked with green squares. 
Figure 15 presents the results of optimisation on the in-plane tilting angle and the tool depth for all field data sets. We observe that over the channel itself and away from its edges, where we dominantly expect horizontal layering, the recovered angles are small. On the other hand, we observe a very large optimal angle at the left edge of the channel, i.e., at $18 \mathrm{~m}$. We attribute that to structural reasons due to the location of the measurement and the lateral contrast from the surrounding soil to the channel material. The measurement at $18 \mathrm{~m}$ is situated directly over the left boundary of the palaeochannel, which we interpret as the cut bank of the stream, with a clear and abrupt change in resistivity. This results in a rather clear division between $\varphi=0^{\circ}$ and $\varphi=180^{\circ}$ here, since on the left side we mainly see the coarser sediments outside of the channel, while on the right side we mainly encounter channel sediments. At $38 \mathrm{~m}$, on the right edge of the channel, the picture is not as clear as that, but the recovered tilting angle is still larger than for most other locations. It is interpreted as the slip-off slope, where we see a more gradual change from channel sediments to river bank sediments in the surface profile. It could also be interpreted as a second channel structure overlaying the main channel. Still, we expect a lateral change in resistivity in the data. From the optimisation result at $18 \mathrm{~m}$, we conclude that if the resistivity is different over an extended area on one side of the tool, our optimisation parameter fails to recover the true tilting angle. The prerequisite for the optimisation is a horizontal layering. The coarse structure can be checked in advance by a surface ERT measurement, for making sure that the soil is horizontally layered. Since the result for the b2s data at $18 \mathrm{~m}$ profile length is questionable, we do not use an optimised tilting angle for this data set, but assume a straight tool in this case and only use the optimised depth in the inversion. We determine the depth in the next step for all b2s data. For this optimisation, we need to invert the data, scanning a range of tool depths around the depth measured in the field. All relevant inversion settings are listed in table 1, i.e., the depth, tilting $\theta$ (positive values mean $\varphi=0^{\circ}$ and negative values equal $\varphi=180^{\circ}$ ), regularisation strength $\lambda$, and the fit of the final model, represented by the relative RMS error and the goodness of fit ( $\chi^{2}$, equals 1 for a perfect fit) before and after the optimisation. We observe on the right side of figure 15 that the final value of the objective function develops in a systematic way for most of the data, resulting in a minimum around a specific tool depth. 

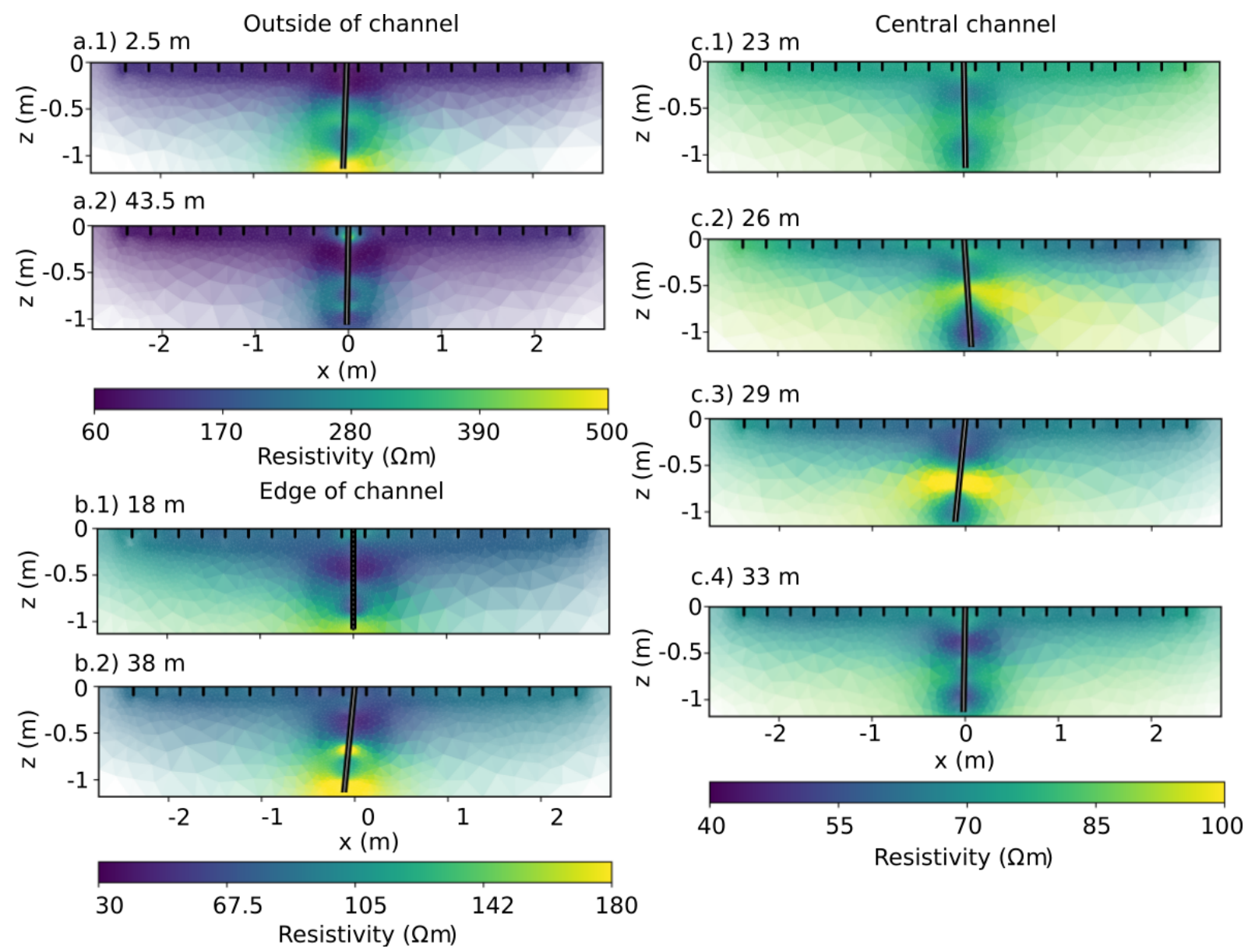

Figure 16: b2s inversion results after angle and depth optimisation in a) the left part of the surface profile, i.e., $2.5 \mathrm{~m}$ and $43.5 \mathrm{~m}, \mathrm{~b}$ ) the central part of the profile, i.e., $18 \mathrm{~m}$ and $38 \mathrm{~m}$, and c) the right part of the profile, i.e., $23 \mathrm{~m}, 26 \mathrm{~m}, 29 \mathrm{~m}, 33 \mathrm{~m}$. The colour scale is adjusted to the different parts of the surface profile and the models are shaded with the coverage. 
Table 1: Inversion settings before and after the optimisation for tilt and depth. Negative tilting angles signify $\varphi=180^{\circ}$.

\begin{tabular}{l|c|c|c|c|c|c|c|c} 
profile meter & 2.5 & 18 & 23 & 26 & 29 & 33 & 38 & 43.5 \\
\hline \hline initial depth $(\mathrm{cm})$ & 8 & 11.7 & 21.7 & 23.7 & 23.7 & 18.7 & 21.7 & 8 \\
\hline optimised depth $(\mathrm{cm})$ & 15 & 9 & 15 & 17 & 12 & 14 & 15 & 7 \\
\hline optimised tilting $\theta\left(^{\circ}\right)$ & -2 & 0 & 1 & 4 & -6 & -1 & -6 & -1 \\
\hline smoothing $(\lambda)$, not optimised & 150 & 200 & 500 & 250 & 150 & 250 & 400 & 80 \\
\hline RMS $(\%) / \chi^{2}$, not optimised & $4.76 / 1.19$ & $4.76 / 1.2$ & $4.43 / 1.0$ & $4.50 / 1.0$ & $4.81 / 0.96$ & $4.41 / 0.99$ & $4.55 / 1.05$ & $4.73 / 1.18$ \\
\hline smoothing $(\lambda)$, optimised & 150 & 200 & 800 & 300 & 500 & 400 & 200 & 80 \\
\hline RMS $(\%) / \chi^{2}$, optimised & $4.72 / 1.03$ & $4.32 / 0.99$ & $4.23 / 0.92$ & $4.26 / 0.93$ & $4.83 / 1.18$ & $4.53 / 1.07$ & $4.49 / 0.98$ & $4.46 / 1.04$ \\
\hline
\end{tabular}

We show the b2s models after the optimisation in figure 16 . The b2s models are able to recover very sharp layer boundaries and the recovered resistivity contrasts are partially very small. Following our findings for synthetic data, we conclude that the recovered layer depths and resistivities are more accurate after the optimisation. The b2s results are consistent in terms of recovered layers over the whole surface profile. Outside the channel (figure 16 a), we have low resistivities in the topmost layer, which is between $30 \mathrm{~cm}$ and $50 \mathrm{~cm}$ thick. The high resistivity around the top of the borehole tool at $43.5 \mathrm{~m}$ seems to be either an artefact, possibly from air around the tool, or a small-scale anomaly like a stone. Below the top layer, the resistivity increases, which is best visible at $2.5 \mathrm{~m}$, where we can clearly identify four layers. At the edge of the palaeochannel (figure $16 \mathrm{~b}$ ), we can also discriminate several layers. A topmost layer of about $20 \mathrm{~cm}$ thickness with a resistivity of about $80 \Omega \mathrm{m}$ is followed by a lower resistivity layer with approximately $30 \Omega \mathrm{m}$ resistivity and a thickness of $20 \mathrm{~cm}$. Below, the resistivity increases to about $150 \Omega \mathrm{m}$ to $180 \Omega \mathrm{m}$, with some sub-layers. For the central part (figure $16 \mathrm{c}$ ), we see a top layer of about $15 \mathrm{~cm}$ to $20 \mathrm{~cm}$ thickness, followed by a less resistive layer below and then a thin layer with increased resistivity, below which the resistivity drops again. The high resitivity layer with approximately $100 \Omega \mathrm{m}$ from about $50 \mathrm{~cm}$ to $70 \mathrm{~cm}$ depth develops around the 26 $\mathrm{m}$ mark and is clearly visible at $29 \mathrm{~m}$ profile length, but has faded again at $33 \mathrm{~m}$. Comparing the final results with the models before the optimisation (figure 14), we see subtle differences outside and over the edges of the palaeochannel. Mainly, the layer boundaries are slightly shifted due to the depth optimisation, and the layer boundaries become sharper after the optimisation. The same is true for the measurements above the central palaeochannel, but additionally we see less artefacts after the optimisation. The depth optimisation has a big influence on the circular artefacts, which resulted from the wrong depth assumption, like the ones visible at $29 \mathrm{~m}$ profile length before the optimisation (figure 14 d.3), which are gone after the optimisation (figure 16 c.3). We appreciate the resolution capabilities of the b2s array, which go down to a vertical spatial resolution of about $10 \mathrm{~cm}$ at the tool surface and to resistivity contrasts of about $5 \Omega \mathrm{m}$ for our data.

\section{Summary and discussion}

We study the influence of borehole-related effects on small-scale b2s ERT and develop approaches to mitigate them. Specifically, we search for the correct placement of the borehole tool regarding its depth and tilt. A tool tilting within the sensitive plane as well as a wrong depth assumption have a considerable influence on the data and inverted models. The effect is already visible at small tilting angles $\theta$ and small depth shifts. On the other hand, the portion of the tilting out of the sensitive plane, i.e., at angles $\varphi$ between $0^{\circ}$ and $180^{\circ}$ or between $180^{\circ}$ and $360^{\circ}$, has a negligible influence on the data and can be ignored, by only searching for the angle $\theta$, projected onto the sensitive plane. We suggest mitigation approaches that correct the tool tilt and depth using the measured data and their inversion, respectively. We do not need to keep the same mesh, as would be necessary for a combined inversion on resistivity and positions, making our 
approach easily applicable to models with 3D electrodes, as shown in this study.

Our metric for deriving the angle of installation relies on the directional sensitivity of the surface electrode array surrounding the borehole tool, and does not allow an optimisation of the tilting out of the sensitive plain, which however is negligible. The metric is not completely independent of the surrounding resistivities, since the sensitivity around the tool bottom is still slightly influenced by the surface electrodes and therefore does not perfectly map one-sided anomalies to the other sides of the tool. Furthermore, for larger lateral contrasts in resistivity on the left and right side of the tool, e.g. for lateral boundaries in the soil, the criterion fails to recover the tool angle. In predominantly layered soils the criterion is easy to apply to any kind of b2s ERT data, regardless of the used electrode model.

Our depth optimisation is also not perfectly independent from the subsurface resistivity distribution, its accuracy decreases especially in the case of large resistivity contrasts in adjacent layers. However, we consider it a valid and easy to use, although not particularly computationally efficient, approach for determining the unknown depth of a borehole electrode array. Further research should go in the direction of a joint inversion on resistivity and electrode positions, possibly utilising fixed points in the model, where the resistivity is calculated consistently, independent from the changing mesh with CEM electrodes.

We additionally account for a borehole filling surrounding the borehole tool for electrical coupling to the ground. When considering the borehole mud accurately by including it in a decoupled borehole region and inverting on its single resistivity, we can eliminate the influence of the mud on the model surrounding the borehole.

Subsequently, we combine all mitigations and demonstrate our correction approaches for $8 \mathrm{~b} 2 \mathrm{~s}$ ERT field measurements, whose positions we chose based on a standard surface ERT result. As no reciprocal data were acquired with the single-channel instrument Lippmann 4-Point light, we developed an error model based on the stacking errors and applied it in the inversion. For this, we used reciprocal data errors from a measurement with the borehole tool alone for determining a base error level for our data. The resulting b2s data errors are reasonable and result in well-fitted, smooth models, which should work well for most layered soils, where the soil type and texture does not change abruptly.

With our b2s measurements, we are able to resolve fine layering, absent from the surface result over the same structure. After correcting for the borehole effects, we get accurate depths for the layers and avoid artefacts. The presented methodology of b2s ERT measurements can be a valuable tool for characterising layered soils. Soil core analysis is a great addition for verifying the models with a "ground truth". However, fine soil layering can be difficult to identify visually in the field, since a discrimination between layers in terms of colour, texture, and saturation might be hard to recognise, and the soil can also be altered by the augering. Using b2s ERT data ensures an identification of all present layers with a resistivity contrast. The method becomes even more promising if extended to time-lapse measurements, where the saturation of different layers can be monitored over a period of time at the same spot. This can be a next step in expanding the b2s methodology.

\section{Acknowledgments}

Special thanks go to Lothar Ahrensmeier for manufacturing the borehole tool and helping with the measurements. The field data in this work were acquired within the framework of Transregional Collaborative Research Centre 32 (SFB-TR32 "Patterns in Soil-Vegetation-Atmosphere 
Systems: Monitoring, Modelling and Data Assimilation"), funded by the German Research Foundation (DFG).

\section{References}

Brogi, C., Huisman, J. A., Pätzold, S., von Hebel, C., Weihermüller, L., Kaufmann, M. S., van der Kruk, J., \& Vereecken, H., 2018. Large-scale soil mapping using multi-configuration EMI and supervised image classification, Geoderma, 335, 133-148.

Brogi, C., Huisman, J., Herbst, M., Weihermüller, L., Klosterhalfen, A., Montzka, C., Reichenau, T., \& Vereecken, H., 2020. Simulation of spatial variability in crop leaf area index and yield using agroecosystem modeling and geophysics-based quantitative soil information, Vadose Zone Journal.

Doetsch, J., Coscia, I., Greenhalgh, S. A., Linde, N., Green, A. G., \& Günther, T., 2010. The borehole-fluid effect in electrical resistivity imaging, Geophysics, 75(4).

Fatichi, S., Or, D., Walko, R., Vereecken, H., Young, M. H., Ghezzehei, T. A., Hengl, T., Kollet, S., Agam, N., \& Avissar, R., 2020. Soil structure is an important omission in earth system models, Nature Communications, 11(522).

Friedel, S., 2003. Resolution, stability and efficiency of resistivity tomography estimated from a generalized inverse approach, Geophysical Journal International, 153, 305-316.

Greinert, A., 2015. The heterogeneity of urban soils in the light of their properties, Soils and Sediments in Urban and Mining Areas, 15, 1725-1737.

Günther, T., Rücker, C., \& Spitzer, K., 2006. Three-dimensional modelling and inversion of dc resistivity data incorporating topograpy - II. Inversion, Geophysical Journal International, 166, 506-517.

Kollet, S. J., 2009. Influence of soil heterogeneity on evapotranspiration under shallow water table conditions: transient, stochastic simulations, Environmental Research Letters, 4.

LaBrecque, D. J. \& Yang, X., 2001. Difference Inversion of ERT Data: a Fast Inversion Method for 3-D In Situ Monitoring, Journal of Environmental and Engineering Geophysics, 6(2), i-106.

Nezhad, M. M., Javadi, A. A., \& Abbasi, F., 2011. Stochastic finite element modelling of water flow in variably saturated heterogeneous soils, International Journal for Numerical and Analytical Methods in Geomechanics, 35, 1389-1408.

Ochs, J. \& Klitzsch, N., 2020. Considerations regarding small-scale surface and borehole-tosurface electrical resistivity tomography, Journal of Applied Geophysics, $\mathbf{1 7 2}$.

Oldenborger, G. A., Routh, P. S., \& Knoll, M. D., 2005. Sensitivity of electrical resistivity tomography data to electrode position errors, Geophysical Journal International, 163, 1-9.

Parsekian, A., Claes, N., Singha, K., Minsley, B., Carr, B., Voytek, E., Harmon, R., Kass, A., Carey, A., Thayer, D., \& Flinchum, B., 2017. Comparing measurement response and inverted results of electrical resistivity tomography instruments, Journal of Environmental and Engineering Geophysics.

Pätzold, S., Mertens, F. M., Bornemann, L., Koleczek, B., Franke, J., Feilhauer, H., \& Welp, G., 2008. Soil heterogeneity at the field scale: a challenge for precision crop protection, Precision Agriculture, 9, 367-390. 
Pätzold, S., Hbirkou, C., Dicke, D., Gerhards, R., \& Welp, G., 2020. Linking weed patterns with soil properties: a long-term case study, Precision Agriculture, 21, 569-588.

Rosenfeld, C. E., Chaney, R. L., Tappero, R. V., \& Martínez, C. E., 2017. Microscale investigations of soil heterogeneity: Impacts on zinc retention and uptake in zinc-contaminated soils, Journal of Environmental Quality, 46, 373-383.

Rücker, C. \& Günther, T., 2011. The simulation of finite ERT electrodes using the complete electrode model, Geophysics, 76(4), F227-F238.

Rücker, C., Günther, T., \& Wagner, F. M., 2017. pyGIMLi: An open-source library for modelling and inversion in geophysics, Computers and Geosciences, 109, 106-123.

Rudolph, S., Van der Kruk, J., von Hebel, C., Ali, M., Herbst, M., Montzka, C., Pätzold, S., Robinson, D. A., Vereecken, H., \& Weihermüller, L., 2015. Linking satellite derived lai patterns with subsoil heterogeneity using large-scale ground-based electromagnetic induction measurements, Geoderma, 241-242, 262-271.

Tso, C. M., Kuras, O., Wilkinson, P. B., Uhlemann, S., Chambers, J. E., Meldrum, P. I., Graham, J., Sherlock, E. F., \& Binley, A., 2017. Improved characterisation and modelling of measurement errors in electrical resistivity tomography (ERT) surveys, Journal of Applied Geophysics, 146, 103-119.

Vanella, D., Cassiani, G., Busato, L., Boaga, J., Barbagallo, S., Binley, A., \& Consoli, S., 2018. Use of small scale electrical resistivity tomography to identify soil-root interactions during deficit irrigation, Journal of Hydrology, 556, 310-324.

Verdet, C., Anguy, Y., Sirieix, C., Clément, R., \& Gaborieau, C., 2018. On the effect of electrode finiteness in small-scale electrical resistivity imaging, Geophysics, 83(6), EN39-EN52.

von Hebel, C., Rudolph, S., Mester, A., Huisman, J. A., Kumbhar, P., Vereecken, H., \& van der Kruk, J., 2014. Three-dimensional imaging of subsurface structural patterns using quantitative large-scale multiconfiguration electromagnetic induction data, Water Resources Research, $\mathbf{5 0}(3), 2732-2748$.

von Hebel, C., Matveeva, M., Verweij, E., Rademske, P., Kaufmann, M. S., Brogi, C., Vereecken, H., Rascher, U., \& van der Kruk, J., 2018. Understanding soil and plant interaction by combining ground-based quantitative electromagnetic induction and airborne hyperspectral data, Geophysical Research Letters, 45(15), 7571-7579.

Wagner, F., Bergmann, P., Rücker, C., Wiese, B., Labitzke, T., Schmidt-Hattenberger, C., \& Maurer, H., 2015. Impact and mitigation of borehole related effects in permanent crosshole resistivity imaging: An example from the Ketzin $\mathrm{CO}_{2}$ storage site, Journal of Applied Geophysics, 123, 102-111.

Weihermüller, L., Huisman, J. A., Lambot, S., Herbst, M., \& Vereecken, H., 2007. Mapping the spatial variation of soil water content at the field scale with different ground penetrating radar techniques, Journal of Hydrology, 340(3-4), 205-216.

Zhou, B. \& Dahlin, T., 2003. Properties and effects of measurement errors on 2d resistivity imaging surveying, Near Surface Geophysics, 2003, 105-117. 